TRANSACTIONS OF THE

AMERICAN MATHEMATICAL SOCIETY

Volume 363, Number 5, May 2011, Pages 2305-2339

S 0002-9947(2010)05167-0

Article electronically published on November 30, 2010

\title{
RULED LAGRANGIAN SUBMANIFOLDS OF THE 6-SPHERE
}

\author{
JASON D. LOTAY
}

\begin{abstract}
This article sets out to serve a dual purpose. On the one hand, we give an explicit description of the Lagrangians in the nearly Kähler 6-sphere $\mathcal{S}^{6}$ which are ruled by circles of constant radius using 'Weierstrass formulae'. On the other hand, we recognise all known examples of these Lagrangians as being ruled by such circles. Therefore, we describe all families of Lagrangians in $\mathcal{S}^{6}$ whose second fundamental form satisfies natural pointwise conditionsthe so-called 'second order families'.
\end{abstract}

\section{INTRODUCTION}

The 6 -sphere $\mathcal{S}^{6}$ inherits a nearly Kähler structure from its natural inclusion in the imaginary octonions. It is thus endowed with an almost symplectic structure, given by a non-degenerate 2 -form $\omega$ which is not closed. We may define Lagrangian submanifolds of $\mathcal{S}^{6}$ as 3 -dimensional submanifolds on which $\omega$ vanishes. Surprisingly, Lagrangians in $\mathcal{S}^{6}$ are minimal and real analytic. They are also related to calibrated 4-dimensional submanifolds of $\mathbb{R}^{7}$ known as coassociative 4 -folds and are analogues of special Lagrangian submanifolds of $\mathbb{C}^{3}$.

1.1. Motivation. Lagrangian submanifolds of $\mathcal{S}^{6}$ have been studied by many authors, and several families of explicit examples are known. The homogeneous examples are classified in [22] and the constant curvature examples in [9]. Some of these examples have been given explicit descriptions in [7. Lagrangians satisfying certain curvature conditions are classified in 6] and 8. Ruled Lagrangian submanifolds in $\mathcal{S}^{6}$ are equivalent to coassociative cones in $\mathbb{R}^{7}$ ruled by 2 -planes, which are studied in 12 and by the author in 20]. A special family of ruled Lagrangians is analysed in 25 .

The study of second order families of submanifolds associated with special holonomy was begun by Bryant 3 . (for special Lagrangian 3-folds in $\mathbb{C}^{3}$ ) and continued in [11] and [16]. Bryant [3, §3] classifies all special Lagrangian 3-folds whose second fundamental form has a pointwise symmetry and gives a characterisation of ruled examples. However, he does not explicitly describe the ruled family, and he remarks that there cannot be a Weierstrass formula for the general ruled special Lagrangian 3-fold. Ruled special Lagrangians in $\mathbb{C}^{3}$ are also studied in [17], and the analogous situation in $\mathbb{R}^{7}$, for calibrated submanifolds called associative 3 -folds, is researched by the author in 19 .

Received by the editors August 22, 2008.

2010 Mathematics Subject Classification. Primary 53B20, 53B25.

(C)2010 American Mathematical Society 
The full classification of Lagrangians in $\mathcal{S}^{6}$ whose second fundamental form has a non-trivial stabilizer is given in [26], though the exposition is rather brief. Here we give more detail and a new perspective on this survey.

1.2. Summary. The culmination of the work in this article is paraphrased in Theorems 1.1 and 1.2 below. Recall that the 6 -sphere is endowed with an almost complex structure, and thus we can define pseudoholomorphic curves $\Sigma$ in $\mathcal{S}^{6}$. If $\Sigma$ is nontotally geodesic we can define its first and second normal bundles $N_{1} \Sigma$ and $N_{2} \Sigma$ respectively, as well as its full normal bundle $N \Sigma$. Given any pseudoholomorphic curve $\Sigma$ we can construct a tube in $N \Sigma$ which is a 3 -dimensional submanifold of $\mathcal{S}^{6}$ ruled by circles of constant radius. A special family of pseudoholomorphic curves are those with null-torsion. For formal definitions we refer the reader to $\$ 2$ \&4

We shall denote a totally geodesic $n$-sphere in $\mathcal{S}^{6}$ by $\mathcal{S}^{n}$, for $n<6$. We say that a submanifold of $\mathcal{S}^{6}$ is linearly full if it is not contained in any $\mathcal{S}^{n}$ in $\mathcal{S}^{6}$ for $n<6$.

Theorem 1.1. Let $L$ be a Lagrangian in $\mathcal{S}^{6}$ and let $h_{L}$ be its second fundamental form.

(a) $L$ is not linearly full if and only if $L$ is the Hopf lift in $\mathcal{S}^{5}$ of a holomorphic curve in $\mathbb{C P}^{2}$ as in Example 6.11. Moreover, $L$ is ruled by geodesic circles and $h_{L}$ has a pointwise symmetry.

(b) $L$ is linearly full and $h_{L}$ admits a pointwise symmetry if and only if $L$ is locally either a tube in $N_{2} \Sigma$ ruled by geodesic circles over a pseudoholomorphic curve $\Sigma \neq \mathcal{S}^{2}$ or a tube ruled by circles of radius $\frac{2}{3}$ over a null-torsion pseudoholomorphic curve $\Sigma$, which is in $N_{2} \Sigma$ if $\Sigma \neq \mathcal{S}^{2}$.

Note. We can define a Lagrangian tube about $\mathcal{S}^{2}$ ruled by circles of radius $\frac{2}{3}$, though we can no longer distinguish the first and second normal bundles.

Theorem 1.2. Let $L$ be a linearly full Lagrangian in $\mathcal{S}^{6}$ and let $h_{L}$ denote its second fundamental form.

(a) Suppose $h_{L}$ does not admit a pointwise symmetry. Then $L$ is ruled by geodesic circles if and only if $L$ is locally a tube about a pseudoholomorphic curve $\Sigma \neq \mathcal{S}^{2}$, defined using a holomorphic curve in a $\mathbb{C P}^{1}$-bundle over $\Sigma$ as in Example 7.4 .

(b) $L$ is ruled by non-geodesic circles of constant radius if and only if $L$ is locally a tube about a null-torsion pseudoholomorphic curve $\Sigma$ (in $N_{2} \Sigma$ when $\Sigma \neq \mathcal{S}^{2}$ ) and the ruling circles have radius $\frac{2}{3}$. Moreover, $h_{L}$ admits a pointwise symmetry.

We begin in $₫ 2$ by defining the nearly Kähler structure on the 6 -sphere and the submanifolds associated with this structure. In $\$ 3$ we introduce the fundamental cubic of a Lagrangian in $\mathcal{S}^{6}$, defined by its second fundamental form, and derive two presentations of the structure equations of $\mathrm{G}_{2}$ which are adapted to the Lagrangian and pseudoholomorphic geometries. In 4 we define ruled Lagrangians and characterise them using CR-holomorphic curves in the space of geodesic circles in $\mathcal{S}^{6}$. We also define tubes about surfaces in $\mathcal{S}^{6}$, which provide examples of ruled 3-dimensional submanifolds of $\mathcal{S}^{6}$.

In $\$ 5$ we start by studying the possible pointwise symmetries of the fundamental cubic. We then explicitly describe the Riemann curvature tensors associated with the induced metrics on Lagrangian submanifolds of $\mathcal{S}^{6}$. In $\$ 6$ we classify the 
Lagrangians in $\mathcal{S}^{6}$ whose fundamental cubic admits a pointwise symmetry. Furthermore, we recognise these examples as Hopf lifts of holomorphic curves or locally as tubes about pseudoholomorphic curves. Theorem 1.1 follows from these considerations.

In $\$ 7$ we give an explicit description of the general ruled Lagrangian. We also classify the Lagrangians that are ruled by geodesic circles. Finally, in 88 , we show that the Lagrangians which are ruled by non-geodesic circles of constant radius have already been described in $\$ 6$. These results prove Theorem 1.2

Note. In this article, we shall occasionally use the theory of exterior differential systems (EDS). We will adopt the standard notion of local dependence of the solutions to an involutive system on $m$ functions of $n$ variables when the last non-zero Cartan character is $s_{n}=m$. The author first came to understand the material discussed in this article through EDS analysis and thought it useful to include some of these considerations. However, it is not essential for the reader to be familiar with EDS in order to understand the paper.

\section{Submanifolds of the Nearly Kähler 6-SPhere}

We shall view the 6-sphere as a nearly Kähler 6-manifold, which we now define.

Definition 2.1. Let $(M, g, J, \omega)$ be an almost Hermitian 6-manifold. That is, $g$ is a Riemannian metric on the almost complex 6-manifold $M, J$ is the almost complex structure preserved by $g$, and $\omega$ is the associated (non-degenerate) Hermitian $(1,1)$ form. The manifold $M$ is nearly Kähler if there exists a nowhere vanishing $(3,0)$ form $\Omega$ on $M$ and a non-zero real constant $\lambda$ such that

$$
\mathrm{d} \omega=3 \lambda \operatorname{Re} \Omega \quad \text { and } \quad \mathrm{d} \operatorname{Im} \Omega=-2 \lambda \omega \wedge \omega .
$$

Equivalently, the $(2,1)$-tensor $G(X, Y)=\nabla_{X}(J) Y$, for vector fields $X, Y$ on $M$, is skew-symmetric and non-zero, where $\nabla$ is the Levi-Civita connection of $g$.

Nearly Kähler 6-manifolds have structure group SU(3). Some authors allow the possibility that $\lambda=0$ with $\mathrm{d} \operatorname{Re} \Omega=0$, which we will see in Definition 2.7 is equivalent to including Calabi-Yau 3-folds. Excluding this case means that we have defined what other authors call strictly nearly Kähler 6-manifolds.

It is well known that a 7-dimensional cone has holonomy $\mathrm{G}_{2}$ if and only if its link is nearly Kähler. Thus, $\mathrm{G}_{2}$ and 6-dimensional nearly Kähler geometry are intimately intertwined. In particular, the nearly Kähler structure on $\mathcal{S}^{6}$ is induced by the $G_{2}$ structure on the imaginary octonions $\operatorname{Im} \mathbb{O}$, recalling that $G_{2}$ is the automorphism group of the cross product algebra of $\operatorname{Im} \mathbb{O}$. It is therefore illuminating to briefly discuss distinguished submanifolds of $\operatorname{Im} \mathbb{O}$.

Definition 2.2. Let $g_{0}$ be the Euclidean metric on $\operatorname{Im} \mathbb{O}$ and let $\times$ denote the octonionic cross product. We can define $\mathrm{G}_{2}$ as the stabilizer in $\mathrm{GL}(\mathrm{Im} \mathbb{O}$ ) of a 3 -form $\varphi_{0}$ on $\operatorname{Im} \mathbb{O}$ which encodes the octonionic cross product via

$$
\varphi_{0}(u, v, w)=g_{0}(u \times v, w) .
$$

This 3-form is closed and coclosed and, by [14, Theorems IV.1.4 \& IV.1.16], $\varphi_{0}$ and its Hodge dual $* \varphi_{0}$ are calibrations. That is, they are closed forms which satisfy $\left.\varphi_{0}\right|_{U} \leq \operatorname{vol}_{U}$ and $\left.* \varphi_{0}\right|_{V} \leq \operatorname{vol}_{V}$, where $U$ and $V$ are oriented tangent 3- and 4planes, respectively. Submanifolds calibrated with respect to $\varphi_{0}$ and $* \varphi_{0}$, i.e. those 
on which they restrict to be volume forms, are called associative 3-folds and coassociative 4 -folds, respectively. We can, by the work in [14, §IV], equivalently define coassociative 4 -folds as the oriented 4-dimensional submanifolds of $\operatorname{Im} \mathbb{O}$ on which $\varphi_{0}$ vanishes (up to a choice of orientation).

For more details on calibrated geometry and these submanifolds, we recommend 13 and 18 .

We now define the nearly Kähler structure on $\mathcal{S}^{6}$.

Definition 2.3. Embedding $\mathcal{S}^{6} \hookrightarrow \operatorname{Im} \mathbb{O}$ as the unit sphere, we endow $\mathcal{S}^{6}$ with the round metric $g$ and identify $T_{p} \mathcal{S}^{6}$ with the 6-plane in $\operatorname{Im} \mathbb{O}$ orthogonal to $p$. Therefore, we can define a map $J_{p}: T_{p} \mathcal{S}^{6} \rightarrow T_{p} \mathcal{S}^{6}$ via left multiplication: $J_{p}(u)=$ $p \times u$. Elementary octonionic algebra shows that $J_{p}^{2}=-1$, so we have an almost complex structure $J$ on $\mathcal{S}^{6}$.

Notice that, on $\operatorname{Im} \mathbb{O}$, we can write

$$
\varphi_{0}=r^{2} \mathrm{~d} r \wedge \omega+r^{3} \Upsilon
$$

for some 2-form $\omega$ and 3 -form $\Upsilon$ on $\mathcal{S}^{6}$, where $r$ is the radial coordinate on $\operatorname{Im} \mathbb{O}$. Identifying $p \in \mathcal{S}^{6}$ with a unit radial vector, we can use (2) and the definition of $J$ to show that $\omega$ is the non-degenerate $(1,1)$-form associated with $J$.

Let $\Omega=\Upsilon+i * \Upsilon$, where $*$ is the Hodge star on $\mathcal{S}^{6}$. Notice that, on $\operatorname{Im} \mathbb{O}$, the Hodge dual of $\varphi_{0}$ can be written as

$$
* \varphi_{0}=\frac{1}{2} r^{4} \omega \wedge \omega-r^{3} \mathrm{~d} r \wedge * \Upsilon .
$$

Therefore, as $\varphi_{0}$ and $* \varphi_{0}$ are closed, $\mathrm{d} \omega=3 \Upsilon$ and $\mathrm{d} * \Upsilon=-2 \omega \wedge \omega$. Finally, we see that $\Omega$ is a nowhere vanishing (3,0)-form and that $\omega$ and $\Omega$ satisfy (1) for $\lambda=1$. Hence, $\mathcal{S}^{6}$ is a nearly Kähler 6 -manifold.

Remarks. The almost complex structure $J$ on $\mathcal{S}^{6}$ is not integrable. Moreover, the 2 -form $\omega$ is clearly not closed, but it does satisfy $\omega \wedge \mathrm{d} \omega=0$.

Having defined the nearly Kähler structure on the 6-sphere, we can present the class of submanifolds we wish to study.

Definition 2.4. A 3-dimensional submanifold $L$ of $\mathcal{S}^{6}$ is Lagrangian if $\left.\omega\right|_{L} \equiv 0$. Equivalently, $J_{p}\left(T_{p} L\right)=N_{p} L$ for all $p \in L$.

Here we have generalised the notion of a Lagrangian submanifold, usually reserved for symplectic manifolds, to the almost symplectic 6 -sphere. However, since $\mathcal{S}^{6}$ has a nearly Kähler structure, Lagrangians in $\mathcal{S}^{6}$ have more properties than one would expect from the general almost symplectic case.

Remark. Many authors refer to Lagrangians in $\mathcal{S}^{6}$ as totally real 3-dimensional submanifolds of $\mathcal{S}^{6}$.

We now show the connection between Lagrangian geometry in $\mathcal{S}^{6}$ and $\mathrm{G}_{2}$ geometry in $\operatorname{Im} \mathbb{O}$, and give some of its consequences.

Proposition 2.5. A 4-dimensional cone in $\operatorname{Im} \mathbb{O}$ is coassociative if and only if its link in $\mathcal{S}^{6}$ is Lagrangian.

Proof. Recall that a 4-dimensional cone $C$ in $\operatorname{Im} \mathbb{O}$ is coassociative if and only if $\left.\varphi_{0}\right|_{C} \equiv 0$. From (3), we see that $\left.\varphi_{0}\right|_{C} \equiv 0$ if and only if $\omega$ and $\Upsilon$ vanish on its link $L$. However, $\Upsilon$ is a non-zero multiple of $\mathrm{d} \omega$, so $\left.\Upsilon\right|_{L} \equiv 0$ if $\left.\omega\right|_{L} \equiv 0$. 
Since coassociative 4-folds are minimal [14, Theorem II.4.2] (in fact, volume minimizing in their homology class [13, Theorem 7.5]) and are real analytic wherever they are non-singular [18, Theorem 12.1.5], we deduce the following.

Corollary 2.6. Lagrangians in $\mathcal{S}^{6}$ are minimal and real analytic away from their singularities.

Remark. The minimality of Lagrangians in $\mathcal{S}^{6}$ is observed in [9, Theorem 1].

We can also perhaps explain these properties of Lagrangians in $\mathcal{S}^{6}$ by considering them as "special Lagrangian" submanifolds of $\mathcal{S}^{6}$. We now take the time to remind the reader of the definition of special Lagrangian $m$-folds.

Definition 2.7. Let $(M, g, J, \omega)$ be a (complex) $m$-dimensional Kähler manifold, where $g$ is the metric, $J$ is the complex structure and $\omega$ is the Kähler form. We say that $M$ is a Calabi-Yau $m$-fold if $M$ is endowed with a nowhere vanishing $(m, 0)$-form $\Omega$ such that $\mathrm{d} \operatorname{Re} \Omega=\mathrm{d} \operatorname{Im} \Omega=0$.

A real oriented $m$-dimensional submanifold $L$ of a Calabi-Yau $m$-fold $M$ is special Lagrangian (with phase $e^{i \theta}$ ) if $\left.\omega\right|_{L} \equiv 0$ and $\left.\operatorname{Im} e^{-i \theta} \Omega\right|_{L} \equiv 0$. Equivalently, $\operatorname{Re} e^{-i \theta} \Omega$ is a calibration on $M$, and special Lagrangian $m$-folds with phase $e^{i \theta}$ are its calibrated submanifolds; i.e. $L$ satisfies $\left.\operatorname{Re} e^{-i \theta} \Omega\right|_{L}=\operatorname{vol}_{L}$.

If $L$ is a 3 -dimensional submanifold of $\mathcal{S}^{6}$ satisfying $\left.\omega\right|_{L} \equiv 0$, then $\Upsilon=\operatorname{Re} \Omega$ also vanishes on $L$ as $3 \Upsilon=\mathrm{d} \omega$. Thus, $L$ is Lagrangian in $\mathcal{S}^{6}$ if and only if $\left.\omega\right|_{L}=$ $\left.\operatorname{Re} \Omega\right|_{L} \equiv 0$. Notice from (44) that, for any oriented tangent 3-plane $V,-\left.* \Upsilon\right|_{V} \leq$ $\operatorname{vol}_{V}$, since $* \varphi_{0}$ is a calibration. Therefore, $-* \Upsilon=-\operatorname{Im} \Omega$ satisfies the condition to be a calibration on $\mathcal{S}^{6}$, except that it is not closed. Moreover, using (4) in conjunction with Proposition 2.5, we observe that $-\left.* \Upsilon\right|_{L}=-\left.\operatorname{Im} \Omega\right|_{L}=\operatorname{vol}_{L}$ for a Lagrangian $L$ in $\mathcal{S}^{6}$. Therefore, Lagrangians in $\mathcal{S}^{6}$ are, in this sense, "special Lagrangian" (with phase $-i$ ) with respect to the nearly Kähler structure. This leads a few authors to call Lagrangian submanifolds of the 6-sphere "special Lagrangian", though we are disinclined to join them in this notation. That said, we can continue the special Lagrangian analogy as follows.

Proposition 2.8. Let $P$ be a real analytic 2-dimensional submanifold of $\mathcal{S}^{6}$ such that $\left.\omega\right|_{P} \equiv 0$. Locally there exists a Lagrangian submanifold $L$ of $\mathcal{S}^{6}$ containing $P$. Moreover, $L$ is locally unique.

Proof. Let $\omega_{0}$ and $\Omega_{0}$ be the Kähler and holomorphic volume forms associated with the standard Calabi-Yau structure on $\mathbb{C}^{3}$. Using the Cartan-Kähler Theorem one can prove the analogous statement of the proposition [14, Theorem III.5.5] for special Lagrangians (with phase $-i$ ) in $\mathbb{C}^{3}$ by considering the exterior differential system on $\mathbb{C}^{3}$ with a differential ideal generated by $\omega_{0}$ and $\operatorname{Re} \Omega_{0}$. By the comments above, for Lagrangians in $\mathcal{S}^{6}$ we are led to consider the EDS with an ideal generated by $\omega$ and $\Upsilon$. Algebraically the ideals have the same properties, since they are both algebraically generated by a 2 -form and a 3 -form (in the special Lagrangian case because they are both closed, and in the Lagrangian case because $3 \Upsilon=\mathrm{d} \omega$ ). Since the proof using the Cartan-Kähler Theorem only relies on the algebra of the EDS, the proposition follows.

By the proof of Proposition 2.8, special Lagrangians in $\mathbb{C}^{3}$ and Lagrangians in $\mathcal{S}^{6}$ have the same 'local existence' properties: they both depend locally on 2 functions of 2 variables. In contrast, Lagrangians in symplectic 6-manifolds, by Darboux's 
theorem, depend locally on an arbitrary function of 3 variables (and there is no need for real analyticity).

Just as there is interplay between the complex and symplectic geometry of a Kähler manifold, there are connections between the almost complex and almost symplectic geometries of the nearly Kähler 6 -sphere. This leads us to define another distinguished class of submanifolds of $\mathcal{S}^{6}$.

Definition 2.9. A 2-dimensional submanifold $\Sigma$ of $\mathcal{S}^{6}$ is a pseudoholomorphic curve if $\left.\omega\right|_{\Sigma}=\operatorname{vol}_{\Sigma}$. Equivalently, $J_{p}\left(T_{p} \Sigma\right)=T_{p} \Sigma$ for all $p \in \Sigma$.

Remark. There are no almost complex 4-folds in $\mathcal{S}^{6}$ [2, Proposition 4.1].

From (3), we observe that a 3 -dimensional cone in $\operatorname{Im} \mathbb{O}$ is associative if and only if its link in $\mathcal{S}^{6}$ is a pseudoholomorphic curve. We may readily deduce some of the well-known properties of pseudoholomorphic curves. First, they are minimal and real analytic away from their singularities. Second, every real analytic curve in $\mathcal{S}^{6}$ can be locally extended in a locally unique way to a pseudoholomorphic curve. Hence, pseudoholomorphic curves in $\mathcal{S}^{6}$ depend locally on 4 functions of 1 variable.

Remarks. We can define Lagrangian and almost complex submanifolds in any nearly Kähler 6-manifold in the obvious manner. Since the results of this section on submanifolds of $\mathcal{S}^{6}$ follow directly from the definition of a nearly Kähler structure in 6 dimensions and from its relationship with $\mathrm{G}_{2}$ geometry, they can all be naturally generalised for the corresponding submanifolds of arbitrary nearly Kähler 6 -manifolds. However, most of the later results in this article rely heavily on the particular geometry of $\mathcal{S}^{6}$ and so will not immediately generalise.

\section{The structure equations And the Fundamental CUbiC}

In this section we provide the formulae that we require for our calculations later. In particular, we view the frame bundle over $\mathcal{S}^{6}$ as $\mathrm{G}_{2}$, since $\mathcal{S}^{6} \cong \mathrm{G}_{2} / \mathrm{SU}(3)$, and thus give two presentations of the structure equations of $\mathrm{G}_{2}$ adapted to the Lagrangian and pseudoholomorphic curve scenarios. Along the way we introduce the fundamental cubic of a Lagrangian submanifold, which is a useful means of encoding the second fundamental form.

Since the nearly Kähler structure on $\mathcal{S}^{6}$ is defined using the octonions $\mathbb{O}$, it is useful for reference to have the multiplication table for $\mathbb{O}$. Let $\mathbb{O}$ be spanned by 1 and $\left\{\varepsilon_{1}, \ldots, \varepsilon_{7}\right\}$ satisfying the multiplication law below.

\begin{tabular}{r|rrrrrrrr} 
& 1 & $\varepsilon_{1}$ & $\varepsilon_{2}$ & $\varepsilon_{3}$ & $\varepsilon_{4}$ & $\varepsilon_{5}$ & $\varepsilon_{6}$ & $\varepsilon_{7}$ \\
\hline 1 & 1 & $\varepsilon_{1}$ & $\varepsilon_{2}$ & $\varepsilon_{3}$ & $\varepsilon_{4}$ & $\varepsilon_{5}$ & $\varepsilon_{6}$ & $\varepsilon_{7}$ \\
$\varepsilon_{1}$ & $\varepsilon_{1}$ & -1 & $\varepsilon_{3}$ & $-\varepsilon_{2}$ & $\varepsilon_{5}$ & $-\varepsilon_{4}$ & $\varepsilon_{7}$ & $-\varepsilon_{6}$ \\
$\varepsilon_{2}$ & $\varepsilon_{2}$ & $-\varepsilon_{3}$ & -1 & $\varepsilon_{1}$ & $\varepsilon_{6}$ & $-\varepsilon_{7}$ & $-\varepsilon_{4}$ & $\varepsilon_{5}$ \\
$\varepsilon_{3}$ & $\varepsilon_{3}$ & $\varepsilon_{2}$ & $-\varepsilon_{1}$ & -1 & $-\varepsilon_{7}$ & $-\varepsilon_{6}$ & $\varepsilon_{5}$ & $\varepsilon_{4}$ \\
$\varepsilon_{4}$ & $\varepsilon_{4}$ & $-\varepsilon_{5}$ & $-\varepsilon_{6}$ & $\varepsilon_{7}$ & -1 & $\varepsilon_{1}$ & $\varepsilon_{2}$ & $-\varepsilon_{3}$ \\
$\varepsilon_{5}$ & $\varepsilon_{5}$ & $\varepsilon_{4}$ & $\varepsilon_{7}$ & $\varepsilon_{6}$ & $-\varepsilon_{1}$ & -1 & $-\varepsilon_{3}$ & $-\varepsilon_{2}$ \\
$\varepsilon_{6}$ & $\varepsilon_{6}$ & $-\varepsilon_{7}$ & $\varepsilon_{4}$ & $-\varepsilon_{5}$ & $-\varepsilon_{2}$ & $\varepsilon_{3}$ & -1 & $\varepsilon_{1}$ \\
$\varepsilon_{7}$ & $\varepsilon_{7}$ & $\varepsilon_{6}$ & $-\varepsilon_{5}$ & $-\varepsilon_{4}$ & $\varepsilon_{3}$ & $\varepsilon_{2}$ & $-\varepsilon_{1}$ & -1.
\end{tabular}

Then $\operatorname{Im} \mathbb{O}$, the imaginary octonions, is spanned by the $\varepsilon_{j}$. We shall denote the cross product on $\operatorname{Im} \mathbb{O}$ by $\times$, as is standard practice.

Note. Some authors use a basis for $\operatorname{Im} \mathbb{O}$ which has the opposite orientation: the difference in our formulae can be accounted for by a change of sign of $\varepsilon_{7}$. 
3.1. Lagrangian submanifolds. Let $\mathbf{x}: L \rightarrow \mathcal{S}^{6}$ be a Lagrangian immersion and let $g_{L}$ be the induced metric on $L$. At each point $p$ in $L$, let $\left\{\mathbf{e}_{1}(p), \mathbf{e}_{2}(p), \mathbf{e}_{3}(p)\right\}$ be an orthonormal basis for $T_{p} L$ and let $\left\{2 \omega_{1}(p), 2 \omega_{2}(p), 2 \omega_{3}(p)\right\}$ define the dual orthonormal coframe. Notice that $\left\{J \mathbf{e}_{1}(p), J \mathbf{e}_{2}(p), J \mathbf{e}_{3}(p)\right\}$ defines an orthonormal basis for $N_{p} L$. We will implicitly identify $L$ with its image in $\mathcal{S}^{6}$ and identify the tangent and normal vectors at $p \in L$ with their push-forwards in $T_{p} \mathcal{S}^{6} \cong\langle p\rangle^{\perp} \subseteq$ $\operatorname{Im} \mathbb{0}$.

Note. We define $2 \omega_{j}$ to be the dual 1-form to the vector $\mathbf{e}_{j}$ to make our structure equations below look neater.

We now introduce the fundamental cubic of a Lagrangian submanifold.

Definition 3.1. Adopting summation notation, we can locally write the second fundamental form of $L$ as

$$
h_{L}=4 h_{i j k} J \mathbf{e}_{i} \otimes \omega_{j} \omega_{k}
$$

for some totally symmetric tensor of functions $h_{i j k}$ which satisfies $h_{i i k}=0$ as $L$ is minimal. We may therefore define the fundamental cubic $C_{L}$ of $L$ as

$$
C_{L}=8 h_{i j k} \omega_{i} \omega_{j} \omega_{k} .
$$

Thus, $C_{L}$ is a section of $S^{3} T^{*} L$ which encodes the second fundamental form of $L$.

We can realise $C_{L}$ pointwise as a homogeneous harmonic cubic on $\mathbb{R}^{3}$. This picture will be useful from an algebraic standpoint.

We now derive an expression for the structure equations of $G_{2}$ best suited to Lagrangian geometry. The Lie algebra of $\mathrm{G}_{2}, \mathfrak{g}_{2}$, has the following matrix presentation:

$$
\begin{aligned}
& \mathfrak{g}_{2}=\left\{\left(\begin{array}{ccc}
0 & -2 \omega^{\mathrm{T}} & -2 \eta^{\mathrm{T}} \\
2 \omega & \alpha+[\omega] & -\beta-[\eta] \\
2 \eta & \beta-[\eta] & \alpha-[\omega]
\end{array}\right): \omega, \eta \in \mathrm{M}_{3 \times 1}(\mathbb{R}),\right. \\
& \left.\alpha \in \operatorname{Skew}_{3}(\mathbb{R}), \beta \in \operatorname{Sym}_{3}^{0}(\mathbb{R})\right\},
\end{aligned}
$$

where $\operatorname{Sym}_{3}^{0}(\mathbb{R})$ is the space of traceless symmetric $3 \times 3$ real matrices and

$$
\left[(x y z)^{\mathrm{T}}\right]=\left(\begin{array}{ccc}
0 & z & -y \\
-z & 0 & x \\
y & -x & 0
\end{array}\right) .
$$

Let $\mathrm{g}: \mathrm{G}_{2} \rightarrow \mathrm{GL}(7, \mathbb{R})$ be the map taking $\mathrm{G}_{2}$ to the identity component of the Lie subgroup of $\operatorname{GL}(7, \mathbb{R})$ with Lie algebra $\mathfrak{g}_{2}$. Write $g=\left(\mathbf{x} \mathbf{e ~ e}^{\perp}\right)$, where $\mathbf{e}=\left(\mathbf{e}_{1} \mathbf{e}_{2} \mathbf{e}_{3}\right)$ and $\mathbf{e}^{\perp}=\left(\mathbf{e}_{1}^{\perp} \mathbf{e}_{2}^{\perp} \mathbf{e}_{3}^{\perp}\right)$ are in $\mathrm{M}_{7 \times 3}(\mathbb{R})$. Since the Maurer-Cartan form $\phi=\mathrm{g}^{-1} \mathrm{dg}$ takes values in $\mathfrak{g}_{2}$, it can be written as

$$
\phi=\left(\begin{array}{ccc}
0 & -2 \omega^{\mathrm{T}} & -2 \eta^{\mathrm{T}} \\
2 \omega & \alpha+[\omega] & -\beta-[\eta] \\
2 \eta & \beta-[\eta] & \alpha-[\omega]
\end{array}\right)
$$

for some appropriate matrices of 1 -forms $\omega, \eta, \alpha, \beta$.

We can adapt frames on $L$ so that $\mathbf{x}$ is identified with a point in $L$, and $\mathbf{e}$ and $2 \omega$ define an orthonormal frame and coframe for $L$. Thus, we can set $\mathbf{e}^{\perp}=J \mathbf{e}$ and see that $\eta$ vanishes on $L$. From this adaptation, we recognise $\alpha+[\omega]$ as the connection 1-form for the Levi-Civita connection $\nabla^{L}$ of the metric $g_{L}$.

From $\mathrm{dg}=\mathrm{g} \phi$ and the Maurer-Cartan equation $\mathrm{d} \phi+\phi \wedge \phi=0$, we immediately derive the first and second structure equations of $\mathrm{G}_{2}$. 
Proposition 3.2. The first structure of equations of $\mathrm{G}_{2}$ can be written as

$$
\begin{aligned}
\mathrm{d} \mathbf{x} & =2 \mathbf{e} \omega+2 \mathbf{e}^{\perp} \eta ; \\
\mathrm{d} \mathbf{e} & =-2 \mathbf{x} \omega^{\mathrm{T}}+\mathbf{e}(\alpha+[\omega])+\mathbf{e}^{\perp}(\beta-[\eta]) ; \\
\mathrm{d} \mathbf{e}^{\perp} & =-2 \mathbf{x} \eta^{\mathrm{T}}-\mathbf{e}(\beta+[\eta])+\mathbf{e}^{\perp}(\alpha-[\omega]) .
\end{aligned}
$$

On the adapted frame bundle of $L$, these equations become

$$
\begin{aligned}
\mathrm{d} \mathbf{x} & =2 \mathbf{e} \omega ; \\
\mathrm{d} \mathbf{e} & =-2 \mathbf{x} \omega^{\mathrm{T}}+\mathbf{e}(\alpha+[\omega])+J \mathbf{e} \beta ; \\
\mathrm{d} J \mathbf{e} & =-\mathbf{e} \beta+J \mathbf{e}(\alpha-[\omega]) .
\end{aligned}
$$

Proposition 3.3. The second structure equations of $\mathrm{G}_{2}$ are

$$
\begin{aligned}
\mathrm{d} \omega & =-(\alpha+[\omega]) \wedge \omega+(\beta+[\eta]) \wedge \eta ; \\
\mathrm{d} \eta & =-(\beta-[\eta]) \wedge \omega-(\alpha-[\omega]) \wedge \eta ; \\
\mathrm{d} \alpha & =-\alpha \wedge \alpha+\beta \wedge \beta+3 \omega \wedge \omega^{\mathrm{T}}+3 \eta \wedge \eta^{\mathrm{T}} ; \\
\mathrm{d} \beta & =-\alpha \wedge \beta-\beta \wedge \alpha-2 \omega \wedge \eta^{\mathrm{T}}+2 \eta \wedge \omega^{\mathrm{T}}-[\omega] \wedge[\eta]+[\eta] \wedge[\omega] .
\end{aligned}
$$

On the adapted frame bundle of $L$ there exists a fully symmetric tensor of functions $h=h_{i j k}$ such that the structure equations become

$$
\begin{aligned}
\mathrm{d} \omega & =-(\alpha+[\omega]) \wedge \omega ; \\
\beta & =2 h \omega ; \\
\mathrm{d} \alpha & =-\alpha \wedge \alpha+\beta \wedge \beta+3 \omega \wedge \omega^{\mathrm{T}} \\
\mathrm{d} \beta & =-\alpha \wedge \beta-\beta \wedge \alpha .
\end{aligned}
$$

Therefore, on the adapted frame bundle of $L$,

$$
\begin{aligned}
\mathrm{d}(\alpha+[\omega])+(\alpha+[\omega]) \wedge(\alpha+[\omega]) & =4\left(h \omega \wedge h \omega+\omega \wedge \omega^{\mathrm{T}}\right) \quad \text { and } \\
\mathrm{d} h+\left(\left(h \alpha+\frac{1}{2} h[\omega]\right)\right) & =H \omega
\end{aligned}
$$

for some fully symmetric tensor of functions $H=H_{i j k l}$, where $(())$ indicates symmetrisation over the indices; i.e. in summation notation,

$$
((h \alpha))_{i j k}=h_{l i j} \alpha_{k l}+h_{l j k} \alpha_{i l}+h_{l k i} \alpha_{j l} .
$$

Here, $h_{i j k}$ defines the fundamental cubic $C_{L}$ of $L$ as in Definition 3.1 Recalling that the connection 1-form of the metric $g_{L}$ is $\alpha+[\omega]$, the equations (7a) and (7b) can be interpreted as Gauss and Codazzi-like equations. Explicitly, if $R_{i j k l}=\operatorname{Riem}\left(g_{L}\right)$, (7a) and (7b) are equivalent to

$$
R_{i j k l}=\sum_{q}\left(h_{i k q} h_{j l q}-h_{i l q} h_{j k q}\right)+\delta_{i k} \delta_{j l}-\delta_{i l} \delta_{j k} \text { and } \nabla^{L} C_{L} \in \Gamma\left(S^{4} T^{*} L\right) .
$$

These conditions are necessary and sufficient for $\left(L, g_{L}\right)$ to be isometrically embedded as a Lagrangian submanifold of $\mathcal{S}^{6}$ with fundamental cubic $C_{L}$. 
3.2. Pseudoholomorphic curves. Recall that, since we have an almost complex structure $J$ on $\mathcal{S}^{6}$, we can define, at each $p \in \mathcal{S}^{6}, T_{p}^{1,0} \mathcal{S}^{6}=\left\{\mathbf{v} \in T_{p} \mathcal{S}^{6} \otimes_{\mathbb{R}} \mathbb{C}\right.$ : $\left.J_{p} \mathbf{v}=i \mathbf{v}\right\}$. A unitary basis for $T_{p} \mathcal{S}^{6}$ is then really a complex basis for $T_{p}^{1,0} \mathcal{S}^{6}$ whose elements are orthogonal and of length $\frac{1}{\sqrt{2}}$.

Let $\mathbf{u}: \Sigma \rightarrow \mathcal{S}^{6}$ be a pseudoholomorphic curve and let $g_{\Sigma}$ be the induced metric on $\Sigma$. At each point $p \in \Sigma$, we can decompose $\left.T_{p}^{1,0} \mathcal{S}^{6}\right|_{\Sigma}=T_{p}^{1,0} \Sigma \oplus N_{p} \Sigma$. Let $\mathbf{f}_{1}(p)$ span the holomorphic tangent space $T_{p}^{1,0} \Sigma$ and let $\theta_{1}(p)$ be the dual 1-form. Let $N_{p} \Sigma=\left\langle\mathbf{f}_{2}(p), \mathbf{f}_{3}(p)\right\rangle_{\mathbb{C}}$ and let $\left\{\theta_{2}(p), \theta_{3}(p)\right\}$ be the obvious dual 1-forms. We can further choose $\left\{\mathbf{f}_{1}(p), \mathbf{f}_{2}(p), \mathbf{f}_{3}(p)\right\}$ to be a unitary frame for $T_{p} \mathcal{S}^{6}$ at each $p \in \Sigma$, and thus the $\theta_{i}(p)$ define a dual unitary coframe.

Recall that the second fundamental form $h_{\Sigma}$ of $\Sigma$, when evaluated on tangent vectors, takes values in the normal bundle of $\Sigma$ in $\mathcal{S}^{6}$. Thus, at every non-totally geodesic point $p \in \Sigma$ we can define $\left.N_{1} \Sigma\right|_{p}$ to be the subspace of $N_{p} \Sigma$ in which $h_{\Sigma}$ takes values when evaluated on elements of $T_{p}^{1,0} \Sigma$, and further define $\left.N_{2} \Sigma\right|_{p}$ to be the subspace of $N_{p} \Sigma$ orthogonal to $\left.N_{1} \Sigma\right|_{p}$. Hence, if $\Sigma$ is not totally geodesic, $N \Sigma$ decomposes into holomorphic line bundles $N_{1} \Sigma$ and $N_{2} \Sigma$, called the first and second normal bundles. In this case, one could adapt frames so that $\mathbf{f}_{2}$ spans $N_{1} \Sigma$ and $\mathbf{f}_{3}$ spans $N_{2} \Sigma$. However, we refrain from making this choice in general.

We shall need the following cross products, which can be calculated by taking explicit imaginary octonionic representatives for $\mathbf{u}, \mathbf{f}_{1}, \mathbf{f}_{2}$ and $\mathbf{f}_{3}$ :

$$
\begin{gathered}
\mathbf{f}_{1} \times \overline{\mathbf{f}}_{1}=\mathbf{f}_{2} \times \overline{\mathbf{f}}_{2}=\mathbf{f}_{3} \times \overline{\mathbf{f}}_{3}=\frac{i}{2} \mathbf{u} \\
\mathbf{f}_{2} \times \mathbf{f}_{3}=\overline{\mathbf{f}}_{1} ; \quad \mathbf{f}_{3} \times \mathbf{f}_{1}=\overline{\mathbf{f}}_{2} ; \quad \mathbf{f}_{1} \times \mathbf{f}_{2}=\overline{\mathbf{f}}_{3} .
\end{gathered}
$$

We can explicitly define a unitary framing for $\left.T \mathcal{S}^{6}\right|_{\Sigma}$ in a neighbourhood $U \subseteq \Sigma$ of each non-totally geodesic point $p \in \Sigma$ as follows. Let $\mathbf{t}_{1}$ be a unit tangent vector on $U$ and let $\mathbf{t}_{2}=J^{\Sigma} \mathbf{t}_{1}$, where $J^{\Sigma}$ is the almost complex structure on $\Sigma$. Notice that $|h|=\left\|h_{\Sigma}(\mathbf{t}, \mathbf{t})\right\|$ is independent of the choice of unit tangent vector $\mathbf{t}$ on $U$. Therefore, identifying the tangent vectors in $\Sigma$ with their push-forwards in $\left.T \mathcal{S}^{6}\right|_{\Sigma}$, define

$$
\begin{gathered}
\mathbf{n}_{1}=\frac{h_{\Sigma}\left(\mathbf{t}_{1}, \mathbf{t}_{1}\right)}{|h|} ; \quad \mathbf{n}_{2}=\frac{h_{\Sigma}\left(\mathbf{t}_{1}, \mathbf{t}_{2}\right)}{|h|} ; \\
\mathbf{b}_{1}=\mathbf{t}_{1} \times \frac{h_{\Sigma}\left(\mathbf{t}_{1}, \mathbf{t}_{1}\right)}{|h|} ; \quad \mathbf{b}_{2}=\mathbf{t}_{2} \times \frac{h_{\Sigma}\left(\mathbf{t}_{2}, \mathbf{t}_{2}\right)}{|h|} ; \\
\mathbf{t}=\frac{1}{2}\left(\mathbf{t}_{1}-i \mathbf{t}_{2}\right) ; \quad \mathbf{n}=\frac{1}{2}\left(\mathbf{n}_{1}-i \mathbf{n}_{2}\right) ; \quad \mathbf{b}=\frac{1}{2}\left(\mathbf{b}_{1}-i \mathbf{b}_{2}\right) .
\end{gathered}
$$

In this way, $\mathbf{t}$ spans $T^{1,0} U, \mathbf{n}$ spans $N_{1} U$ and $\mathbf{b}$ spans $N_{2} U$.

From [2, Proposition $2.3 \& \S 4$, we may use a complex matrix presentation of $\mathfrak{g}_{2}$ and write the map $\mathrm{g}: \mathrm{G}_{2} \rightarrow \mathrm{GL}(7, \mathbb{C})$ as $\mathrm{g}=(\mathbf{u} \mathbf{f} \overline{\mathbf{f}})$, where $\mathbf{f}=\left(\mathbf{f}_{1} \mathbf{f}_{2} \mathbf{f}_{3}\right) \in$ $\mathrm{M}_{7 \times 3}(\mathbb{C})$, to derive the structure equations of $\mathrm{G}_{2}$. Over $\Sigma$, we recognise $\mathbf{u}$ as a point in $\Sigma$ and $\mathbf{f}$ as a unitary frame for $\left.T \mathcal{S}^{6}\right|_{\Sigma}$. Thus, on the adapted frame bundle over $\Sigma$, we can set $\theta_{2}=\theta_{3}=0$. We deduce the following result.

Proposition 3.4. For a $3 \times 1$ vector of complex-valued 1 -forms $\theta=\left(\theta_{1}, \theta_{2}, \theta_{3}\right)^{\mathrm{T}}$ and $a 3 \times 3$ skew-Hermitian matrix of 1-forms $\kappa=\left(\kappa_{i j}\right)$ satisfying $\operatorname{Tr} \kappa=0$, the 
structure equations of $\mathrm{G}_{2}$ can be written as

$$
\begin{aligned}
\mathrm{d} \mathbf{u} & =-2 i \mathbf{f} \theta+2 i \overline{\mathbf{f}} \bar{\theta} \\
\mathrm{d} \mathbf{f} & =-i \mathbf{u} \bar{\theta}^{\mathrm{T}}+\mathbf{f} \kappa+\overline{\mathbf{f}}[\theta] ; \\
\mathrm{d} \theta & =-\kappa \wedge \theta+[\bar{\theta}] \wedge \bar{\theta} ; \\
\mathrm{d} \kappa & =-\kappa \wedge \kappa+3 \theta \wedge \bar{\theta}^{\mathrm{T}}-\theta^{\mathrm{T}} \wedge \bar{\theta} \mathrm{Id}_{3},
\end{aligned}
$$

where $\operatorname{Id}_{3}$ is the $3 \times 3$ identity matrix.

On the adapted frame bundle of $\Sigma$, there exist holomorphic functions $k_{2}$ and $k_{3}$ such that $\kappa_{21}=k_{2} \theta_{1}$ and $\kappa_{31}=k_{3} \theta_{1}$, and the structure equations become

$$
\begin{aligned}
\mathrm{d} \mathbf{u} & =-2 i \mathbf{f}_{1} \theta_{1}+2 i \overline{\mathbf{f}}_{1} \bar{\theta}_{1} ; \\
\mathrm{d} \mathbf{f}_{1} & =-i \mathbf{u} \bar{\theta}_{1}-\mathbf{f}_{1}\left(\kappa_{22}+\kappa_{33}\right)+k_{2} \mathbf{f}_{2} \theta_{1}+k_{3} \mathbf{f}_{3} \theta_{1} ; \\
\mathrm{d} \mathbf{f}_{2} & =-\bar{k}_{2} \mathbf{f}_{1} \bar{\theta}_{1}+\mathbf{f}_{2} \kappa_{22}+\mathbf{f}_{3} \kappa_{32}-\overline{\mathbf{f}}_{3} \theta_{1} ; \\
\mathrm{d} \mathbf{f}_{3} & =-\bar{k}_{3} \mathbf{f}_{1} \bar{\theta}_{1}-\mathbf{f}_{2} \bar{\kappa}_{32}+\mathbf{f}_{3} \kappa_{33}+\overline{\mathbf{f}}_{2} \theta_{1} ; \\
\mathrm{d} \theta_{1} & =\left(\kappa_{22}+\kappa_{33}\right) \wedge \theta_{1} ; \\
\mathrm{d} \kappa_{22} & =\left(\left|k_{2}\right|^{2}-1\right) \theta_{1} \wedge \bar{\theta}_{1}-\kappa_{32} \wedge \bar{\kappa}_{32} ; \\
\mathrm{d} \kappa_{33} & =\left(\left|k_{3}\right|^{2}-1\right) \theta_{1} \wedge \bar{\theta}_{1}+\kappa_{32} \wedge \bar{\kappa}_{32} ; \\
\mathrm{d}\left(k_{2} \theta_{1}\right) & =-\left(k_{2}\left(2 \kappa_{22}+\kappa_{33}\right)-k_{3} \bar{\kappa}_{32}\right) \wedge \theta_{1} ; \\
\mathrm{d}\left(k_{3} \theta_{1}\right) & =-\left(k_{3}\left(\kappa_{22}+2 \kappa_{33}\right)+k_{2} \kappa_{32}\right) \wedge \theta_{1} ; \\
\mathrm{d} \kappa_{32} & =\bar{k}_{2} k_{3} \theta_{1} \wedge \bar{\theta}_{1}+\left(\kappa_{22}-\kappa_{33}\right) \wedge \kappa_{32} .
\end{aligned}
$$

We can interpret the functions $\left(k_{2}, k_{3}\right)$ as the second fundamental form of the pseudoholomorphic curve. Indeed, by [2, Lemma 4.4], $\left(k_{2}, k_{3}\right)=0$ if and only if $\Sigma$ lies in a totally geodesic $\mathcal{S}^{2}$, which is the intersection of a linear associative 3-plane in $\operatorname{Im} \mathbb{( 1 )}$ with $\mathcal{S}^{6}$. We can observe this ourselves using (11e)-(11g).

Suppose that $\Sigma$ is non-totally geodesic. If we adapt frames further, as suggested earlier, so that $\mathbf{f}_{2}$ and $\mathbf{f}_{3}$ span $N_{1} \Sigma$ and $N_{2} \Sigma$, we find that $k_{3}=0$. Moreover, by [2, Lemma 4.5], there exists a holomorphic function $k_{1}$ such that $\kappa_{32}=k_{1} \theta_{1}$. Following [2], we call $k_{1}$ the torsion of $\Sigma$. The pseudoholomorphic curves with nulltorsion (also called superminimal in the language of integrable systems) exhibit a rich geometry; in fact, every Riemann surface can be immersed as a null-torsion curve in $\mathcal{S}^{6}$ by [2, Theorem 4.10]. It is also straightforward to see from (11) that $\Sigma$ lies in a totally geodesic $\mathcal{S}^{5}$ if and only if the torsion is constant and satisfies $\left|k_{1}\right|=1$.

By [1, Lemma 4.3], pseudoholomorphic curves in $\mathcal{S}^{6}$ split into four types: linearly full and null-torsion; linearly full with non-zero torsion; linearly full in a totally geodesic $\mathcal{S}^{5}$ (and necessarily with non-zero torsion); and totally geodesic.

\section{RULED AND QUASI-RULED LAGRANGIAN SUBMANIFOLDS}

We consider Lagrangians in $\mathcal{S}^{6}$ that are ruled by circles of constant radius. However, we reserve the notation 'ruled' for the case where the circles are geodesics.

Definition 4.1. Let $L$ be a 3 -dimensional submanifold of $\mathcal{S}^{6}$ and let $\lambda \in(0,1]$ be constant. A $\lambda$-ruling of $L$ is a pair $(\Sigma, \pi)$ where $\pi: L \rightarrow \Sigma$ is a smooth fibration of $L$ over a 2 -manifold $\Sigma$ by oriented circles of radius $\lambda$ in $\mathcal{S}^{6}$. We say that $(L, \Sigma, \pi)$ 
is ruled if $(\Sigma, \pi)$ is a 1-ruling of $L$ and that it is quasi-ruled if $(\Sigma, \pi)$ is a $\lambda$-ruling of $L$ for $\lambda \in(0,1)$.

We begin by describing the 'second order' condition on a Lagrangian corresponding to the ruled or quasi-ruled condition.

Lemma 4.2. Let $L$ be a Lagrangian in $\mathcal{S}^{6}$ with a $\lambda$-ruling and let $C_{L}$ be its fundamental cubic. There exists an orthonormal frame $\left\{\mathbf{e}_{1}, \mathbf{e}_{2}, \mathbf{e}_{3}\right\}$ of $L$, with dual coframe $\left\{2 \omega_{1}, 2 \omega_{2}, 2 \omega_{3}\right\}$, such that $\mathbf{e}_{1}$ is the direction of the $\lambda$-ruling and

$$
\begin{aligned}
& C_{L}=C(r, s, a, b) \\
& =r \omega_{1}\left(2 \omega_{1}^{2}-3 \omega_{2}^{2}-3 \omega_{3}^{2}\right)+3 s \omega_{1}\left(\omega_{2}^{2}-\omega_{3}^{2}\right)+a \omega_{2}\left(\omega_{2}^{2}-3 \omega_{3}^{2}\right)+b \omega_{3}\left(3 \omega_{2}^{2}-\omega_{3}^{2}\right),
\end{aligned}
$$

where $r=\frac{4}{\lambda} \sqrt{1-\lambda^{2}}$ and $s, a, b$ are functions. Moreover, if $s=0$ we can choose $b=0$.

Proof. Since $L$ has a $\lambda$-ruling, $h_{L}\left(\mathbf{e}_{1}, \mathbf{e}_{1}\right)=r J \mathbf{e}_{1}$ for some constant $r$. Therefore, it is easy to see that $C_{L}=C(r, s, a, b)$. To determine the relationship between $r$ and $\lambda$, one need only look at the first structure equations (5) for $\omega_{2}=\omega_{3}=0$ :

$$
\mathrm{d} \mathbf{x}=2 \mathbf{e}_{1} \omega_{1} ; \quad \mathrm{d} \mathbf{e}_{1}=-2 \mathbf{x} \omega_{1}+\frac{r}{2} J \mathbf{e}_{1} \omega_{1} ; \quad \mathrm{d} J \mathbf{e}_{1}=\frac{r}{2} \mathbf{e}_{1} .
$$

These are the equations for a circle of radius $4\left(16+r^{2}\right)^{-\frac{1}{2}}$, which must necessarily equal $\lambda$. The formula for $r$ follows. If $s=0$, then we may use the $\mathrm{SO}(2)$ subgroup of $\mathrm{SO}(3)$ that fixes $\mathbf{e}_{1}$ to set $b=0$.

In $₫ 1$, we mentioned the relationship between ruled Lagrangians in $\mathcal{S}^{6}$ and 2-ruled coassociative 4 -folds in $\operatorname{Im} \mathbb{O}$. We now briefly define the latter.

Definition 4.3. Let $N$ be a 4-dimensional submanifold of $\operatorname{Im} \mathbb{O}$. A 2-ruling of $N$ is a pair $(\Sigma, \pi)$ where $\pi: N \rightarrow \Sigma$ is a smooth fibration of $N$ over a 2-manifold $\Sigma$ by oriented affine 2 -planes in $\operatorname{Im} \mathbb{O}$. We say that $(N, \Sigma, \pi)$ is 2 -ruled if $(\Sigma, \pi)$ is a 2-ruling of $N$.

We now make an elementary observation.

Lemma 4.4. A 4-dimensional coassociative cone in $\operatorname{Im} \mathbb{O}$ is 2-ruled if and only if its Lagrangian link in $\mathcal{S}^{6}$ is ruled.

By [12, Proposition 7.2], there is a correspondence between 2-ruled coassociative cones in $\operatorname{Im} \mathbb{O}$ and certain surfaces in the Grassmannian of oriented 2-planes in $\operatorname{Im} \mathbb{O}, \mathrm{Gr}_{+}(2, \operatorname{Im} \mathbb{O})$. Notice that $\mathrm{Gr}_{+}(2, \operatorname{Im} \mathbb{O})$ is naturally isomorphic to the space $\mathcal{C}$ of oriented geodesic circles in $\mathcal{S}^{6}$ : simply identify an oriented 2-plane in $\operatorname{Im} \mathbb{0}$ with its intersection with $\mathcal{S}^{6}$. Therefore, a surface $\phi: \Sigma \rightarrow \mathcal{C}$ can be written as

$$
\phi(p)=\left(\mathbf{v}_{1}(p), \mathbf{v}_{2}(p)\right),
$$

where $\mathbf{v}_{1}, \mathbf{v}_{2}: \Sigma \rightarrow \mathcal{S}^{6}$ are everywhere orthogonal, and so define an oriented basis for a 2 -plane at each point. We then associate a map $\Phi: \Sigma \times[0,2 \pi) \rightarrow \mathcal{S}^{6}$ to $\phi$, whose image is a ruled 3-dimensional submanifold, in the obvious way:

$$
\Phi(p, t)=\mathbf{v}_{1}(p) \cos t+\mathbf{v}_{2}(p) \sin t .
$$

To state our result we need to define almost CR-structures. 
Definition 4.5. An almost CR-structure on a manifold $M$ is a pair $(E, I)$, where $E$ is an even-dimensional subbundle of $T M$ and $I$ is a complex structure map on $E$. An almost CR-structure $(E, I)$ is Levi-flat if for every 1-form $\eta$ on $M$ such that $\left.\eta\right|_{E}=0, \mathrm{~d} \eta$ vanishes on all complex lines in $E$.

Let $M$ be endowed with an almost CR-structure $(E, I)$. A surface $\Sigma$ in $M$ is a CR-holomorphic curve if $T_{p} \Sigma$ is a complex line in $E$ for all $p \in \Sigma$.

Using Lemma 4.4 and [12, Proposition 7.2], we deduce the following.

Proposition 4.6. Let $\mathcal{C}$ be the space of oriented geodesic circles in $\mathcal{S}^{6}$. There is a complex structure I on an 8-plane bundle $E \subseteq T \mathcal{C}$ such that:

(i) $(E, I)$ is a real analytic, Levi-flat, $\mathrm{G}_{2}$-invariant almost CR-structure on $\mathcal{C}$,

(ii) every CR-holomorphic curve $\phi: \Sigma \rightarrow \mathcal{C}$ as in (12) defines a ruled Lagrangian in $\mathcal{S}^{6}$ via $\Phi: \Sigma \times[0,2 \pi) \rightarrow \mathcal{S}^{6}$ as in (13), and

(iii) every ruled Lagrangian $(L, \Sigma, \pi)$ in $\mathcal{S}^{6}$ defines a CR-holomorphic curve in $\mathcal{C}, \phi: \Sigma \rightarrow \mathcal{C}$, where $\phi(p)=\pi^{-1}(p)$.

Remarks. This is the natural analogue of the characterisation of ruled special Lagrangians in $\mathbb{C}^{3}$ given in [3, Theorem 6]. We should stress that our proposition is little more than a repackaging of the material given in [12, Proposition 7.2].

It follows from [12, §8] that CR-holomorphic curves in $\mathcal{C}$ are lifts of pseudoholomorphic curves in $\mathcal{S}^{6}$. We shall show that, in fact, every linearly full ruled or quasi-ruled Lagrangian is locally a tube about a pseudoholomorphic curve defined using holomorphic data.

Definition 4.7. Let $\mathbf{u}: \Sigma \rightarrow \mathcal{S}^{6}$ be an immersed surface. Let $\Pi$ be an oriented 2-plane subbundle of $\mathbf{u}^{*}\left(T \mathcal{S}^{6}\right)$ and $\operatorname{let} \mathcal{U}(\Pi)=\{\mathbf{v} \in \Pi:|\mathbf{v}|=1\}$. Let $\gamma \in\left(0, \frac{\pi}{2}\right]$ be a constant. Define $\mathbf{x}_{\gamma}: \mathcal{U}(\Pi) \rightarrow \mathcal{S}^{6}$ by

$$
\mathbf{x}_{\gamma}(\mathbf{v})=\cos \gamma \mathbf{u}+\sin \gamma \mathbf{v}
$$

When $\mathbf{x}_{\gamma}$ is an immersion, we say that its image is a tube of radius $\gamma$ (in $\Pi$ ) about $\Sigma$. Clearly, a tube of radius $\gamma$ has a $\sin \gamma$-ruling and is thus ruled if $\gamma=\frac{\pi}{2}$ and quasi-ruled otherwise.

Remark. This is a generalisation of the tubes about pseudoholomorphic curves first studied in [10].

\section{The fundamental Cubic and the Gauss equation}

In this section we discuss the possible pointwise symmetries of the fundamental cubic and then analyse the Riemann curvature tensors satisfying the Gauss equation. Though this is strictly more than we require, we feel it is inherently interesting and that it helps expose the links between the symmetries of the fundamental cubic and the curvature conditions studied by other authors.

We remarked earlier that the fundamental cubic $C_{L}$ of a Lagrangian $L$ in $\mathcal{S}^{6}$ naturally defines, at each point, a homogeneous cubic $h=h_{i j k} x_{i} x_{j} x_{k}$ which is harmonic in the variables $\left(x_{1}, x_{2}, x_{3}\right)=(x, y, z)$ on $\mathbb{R}^{3}$. We now exploit this fact, since these cubics on $\mathbb{R}^{3}$ are classified according to their stabilizer in $\mathrm{SO}(3)$ in 3 , Proposition 1].

Proposition 5.1. Let $\mathcal{H}^{3}\left(\mathbb{R}^{3}\right)$ denote the space of homogeneous harmonic cubics on $\mathbb{R}^{3}$. The stabilizer of $h \in \mathcal{H}^{3}\left(\mathbb{R}^{3}\right)$ in $\mathrm{SO}(3)$ is non-trivial if and only if $h$ lies on the $\mathrm{SO}(3)$-orbit of exactly one of the polynomials in Table 1 below. 
TABLE 1. Homogeneous harmonic cubics on $\mathbb{R}^{3}$ with symmetries

\begin{tabular}{|c|c|c|}
\hline Cubic in $\mathcal{H}^{3}\left(\mathbb{R}^{3}\right)$ & Parameter(s) & Stabilizer \\
\hline 0 & & $\mathrm{SO}(3)$ \\
$r x\left(2 x^{2}-3 y^{2}-3 z^{2}\right)$ & $r>0$ & $\mathrm{SO}(2)$ \\
$3 s x\left(y^{2}-z^{2}\right)$ & $s>0$ & $\mathrm{~A}_{4}$ \\
$a y\left(y^{2}-3 z^{2}\right)$ & $a>0$ & $\mathrm{~S}_{3}$ \\
$r x\left(2 x^{2}-3 y^{2}-3 z^{2}\right)+a y\left(y^{2}-3 z^{2}\right)$ & $r, a>0, r \sqrt{2} \neq a$ & $\mathbb{Z}_{3}$ \\
$r x\left(2 x^{2}-3 y^{2}-3 z^{2}\right)+3 s x\left(y^{2}-z^{2}\right)$ & $r, s>0, r \neq s$ & $\mathbb{Z}_{2}$ \\
\hline
\end{tabular}

Remarks. The exclusions $r \sqrt{2} \neq a$ and $r \neq s$ in Table 1 occur for the following reasons. The cubic $r x\left(2 x^{2}-3 y^{2}-3 z^{2}\right)+r \sqrt{2} y\left(y^{2}-3 z^{2}\right)$ lies on the $\mathrm{SO}(3)$-orbit of $3 r \sqrt{3} x\left(y^{2}-z^{2}\right)$, which has an $\mathrm{A}_{4}$-stabilizer, and $r x\left(2 x^{2}-3 y^{2}-3 z^{2}\right)+3 r x\left(y^{2}-z^{2}\right)$ lies on the $\mathrm{SO}(3)$-orbit of $2 r y\left(y^{2}-3 z^{2}\right)$, which has an $\mathrm{S}_{3}$-stabilizer.

We now study whether a given Riemann curvature tensor can arise as a quadratic in the coefficients of a homogeneous harmonic cubic, as specified by the Gauss equation (7a). Since the Riemann curvature tensor is a slightly unwieldy algebraic object, we simplify matters using the following simple definition.

Definition 5.2. Let $R_{a b c d}$ be the Riemann curvature tensor of the metric $g_{L}$ on $L$. For cyclic permutations ( $i j k$ ) of (1 223$)$ define, using the "omitted index" rule, $K_{i i}=R_{j k j k}-1$ and $K_{i j}=R_{j k k i}$. The resulting tensor $K_{a b}$ can be thought of as a $3 \times 3$ symmetric matrix $K$. Define a map from $\mathcal{H}^{3}\left(\mathbb{R}^{3}\right)$ to $\operatorname{Sym}_{3}(\mathbb{R}), h \mapsto K(h)$ by

$$
K(h)_{i i}=\sum_{q} h_{j j q} h_{k k q}-h_{j k q}^{2} \quad \text { and } \quad K(h)_{i j}=\sum_{q} h_{i k q} h_{k j q}-h_{i j q} h_{k k q} ;
$$

i.e. we use the Gauss equation (7a) for $h$. We call this the Gauss map.

Remark. We can recover the Ricci tensor $R_{a b}$ from $K_{a b}$ by the formulae $R_{i i}=$ $K_{j j}+K_{k k}+2$ and $R_{i j}=-K_{i j}$, again using cyclic permutations $(i j k)$ of $\left(\begin{array}{lll}1 & 2 & 3\end{array}\right)$.

This definition leads us to consider the set of $K(h)$ for $h \in \mathcal{H}^{3}\left(\mathbb{R}^{3}\right)$. Notice that $K(0)=0$ and $K(t h)=t^{2} K(h)$ for all $t \in \mathbb{R}$, so the image of the Gauss map is a (1-sided) cone in $\operatorname{Sym}_{3}(\mathbb{R})$. We shall give a description of this cone using a rather "brute force" approach.

As we have the freedom to transform the frame over $L$, we can apply $\mathrm{SO}(3)$ transformations to the source cubic or the target matrix in the Gauss map. In particular, we can diagonalise $K(h)$ to $\operatorname{diag}\left(\lambda_{1}, \lambda_{2}, \lambda_{3}\right)$. We start by studying the case where $K(h)$ has distinct eigenvalues since the calculations here (though still ugly!) are more straightforward.

Proposition 5.3. Let $K=\operatorname{diag}\left(\lambda_{1}, \lambda_{2}, \lambda_{3}\right)$ with $\lambda_{1}>\lambda_{2}>\lambda_{3}$. Let $\sigma(K)=$ $\frac{1}{6}\left((\operatorname{Tr} K)^{2}-\operatorname{Tr} K^{2}\right)$. Then $K=K(h)$ for some $h \in \mathcal{H}^{3}\left(\mathbb{R}^{3}\right)$ as in (14) if and only if $\operatorname{Tr} K<0, \sigma(K)>0$ and $\lambda_{1}^{2} \leq \sigma(K)$.

Proof. If we let $\|h\|^{2}=h_{i j k} h_{i j k}$, using summation notation, then it is easy to calculate that

$$
\operatorname{Tr} K(h)=-\frac{1}{2}\|h\|^{2} .
$$

Therefore $\operatorname{Tr} K(h)<0$, as $K(h)$ is necessarily non-zero. 
The equation $K=K(h)$ has a solution if and only if a quadratic in the coefficients of $h$ has real solutions. Calculation shows that the generic solutions are, for $r$, a real parameter:

$$
\begin{aligned}
h_{331} & =r \\
h_{221} & =\frac{\lambda_{1}-\lambda_{3}}{\lambda_{1}-\lambda_{2}} r ; \\
h_{112} & = \pm\left(\frac{\left(\lambda_{1}-\lambda_{2}\right)^{2}\left(\lambda_{3}^{2}-\sigma(K)\right)-2\left(\lambda_{1}-\lambda_{3}\right)^{3} r^{2}}{2\left(\lambda_{1}-\lambda_{2}\right)^{2}\left(\lambda_{2}-\lambda_{3}\right)}\right)^{\frac{1}{2}} ; \\
h_{332} & =-\frac{\lambda_{1}-\lambda_{2}}{\lambda_{2}-\lambda_{3}} h_{112} ; \\
h_{113} & = \pm\left(\frac{\left(\sigma(K)-\lambda_{2}^{2}\right)+2\left(\lambda_{1}-\lambda_{2}\right) r^{2}}{2\left(\lambda_{2}-\lambda_{3}\right)}\right)^{\frac{1}{2}} ; \\
h_{223} & =\frac{\lambda_{1}-\lambda_{3}}{\lambda_{2}-\lambda_{3}} h_{113} ; \text { and } \\
h_{123} & =0 .
\end{aligned}
$$

Notice that the parameter $r$ is constrained such that if we let

$$
s=2\left(\lambda_{1}-\lambda_{3}\right)^{3}\left(\lambda_{1}-\lambda_{2}\right) r^{2} \geq 0,
$$

then

$$
\left(\lambda_{1}-\lambda_{3}\right)^{3}\left(\lambda_{2}^{2}-\sigma(K)\right) \leq s \leq\left(\lambda_{1}-\lambda_{2}\right)^{3}\left(\lambda_{3}^{2}-\sigma(K)\right) .
$$

The difference in the upper and lower bounds is $\left(\lambda_{2}-\lambda_{3}\right)^{3}\left(\sigma(K)-\lambda_{1}^{2}\right)$, so we must have that $\lambda_{1}^{2} \leq \sigma(K)$ for real solutions to exist.

The condition $\lambda_{1}^{2} \leq \sigma(K)$ clearly forces $\sigma(K) \geq 0$. If $\sigma(K)=0, \lambda_{1}=0$ and

$$
3 \sigma(K)=\lambda_{1} \lambda_{2}+\lambda_{2} \lambda_{3}+\lambda_{3} \lambda_{1}=\lambda_{2} \lambda_{3}=0,
$$

so at least two of the eigenvalues are zero, and we have our required contradiction.

Further, real solutions exist for $r \neq 0$ only if $\lambda_{3}^{2}>\sigma(K)$, and for $r=0$ we need $\lambda_{3}^{2} \geq \sigma(K)$ and $\lambda_{2}^{2} \leq \sigma(K)$. However, we see that

$$
3\left(\lambda_{3}^{2}-\sigma(K)\right)=\lambda_{3}\left(\lambda_{3}-\lambda_{2}\right)+\lambda_{3}\left(\lambda_{3}-\lambda_{1}\right)+\lambda_{3}^{2}-\lambda_{1} \lambda_{2}
$$

Thus, if $\lambda_{1} \lambda_{2} \leq 0$, then $\lambda_{3}^{2}>\sigma(K)$. If $\lambda_{1} \lambda_{2}>0$, then the fact that $\operatorname{Tr} K<0$ forces $\lambda_{3}^{2}>\lambda_{1} \lambda_{2}$; hence $\lambda_{3}^{2}>\sigma(K)$ as well. Thus $\lambda_{3}^{2}>\sigma(K)$ always holds.

The only question left is whether $\lambda_{2}^{2} \leq \sigma(K)$ is an additional constraint. Since $\lambda_{3}=\operatorname{Tr} K-\lambda_{1}-\lambda_{2}$ and $\lambda_{3}^{2}<(\operatorname{Tr} K)^{2}$, because $\sigma(K)>0$, we must have that $\lambda_{1}+\lambda_{2}<0$. Hence $\lambda_{1}^{2}<\lambda_{2}^{2}$, so $\lambda_{2}^{2} \leq \sigma(K)$ implies $\lambda_{1}^{2} \leq \sigma(K)$.

Before stating our next result, we notice from Table 1 that there are two families of cubics $h$ with $\mathbb{Z}_{2}$-stabilizer, given by $r>s$ and $r<s$. This leads to two distinct families of corresponding matrices $K(h)$.

Proposition 5.4. Let $K \in \operatorname{Sym}_{3}(\mathbb{R})$, let $\sigma(K)=\frac{1}{6}\left((\operatorname{Tr} K)^{2}-\operatorname{Tr} K^{2}\right)$ and let $\lambda_{1} \geq \lambda_{2} \geq \lambda_{3}$ be the eigenvalues of $K$. Then $K=K(h)$ for some $h \in \mathcal{H}^{3}\left(\mathbb{R}^{3}\right)$ as in (14) if and only if $\operatorname{Tr} K \leq 0, \sigma(K) \geq 0$ and $\lambda_{1}^{2} \leq \sigma(K)$.

Moreover, the following hold for $K=K(h), h \in \mathcal{H}^{3}\left(\mathbb{R}^{3}\right)$ :

(i) $\operatorname{Tr} K=0$ if and only if $K=h=0$. 
(ii) $\sigma(K)=0$ and $K \neq 0$ if and only if $\lambda_{1}=\lambda_{2}=0>\lambda_{3}$, which occurs if and only if $h$ has an $\mathrm{S}_{3}$-stabilizer.

(iii) $\lambda_{1}^{2}=\sigma(K)>0$ and $K$ has distinct eigenvalues only if $h$ has a $\mathbb{Z}_{2}$-stabilizer.

(iv) $\lambda_{1}^{2}=\sigma(K)>0$ and $K$ has exactly two repeated eigenvalues if and only if $-\frac{1}{5} \operatorname{Tr} K=\lambda_{1}>\lambda_{2}=\lambda_{3}=\frac{3}{5} \operatorname{Tr} K$, which occurs if and only if $h$ has an $\mathrm{SO}(2)$-stabilizer.

(v) $\lambda_{1}^{2}=\sigma(K)>0$ and $K$ has three repeated eigenvalues if and only if $K=$ $-\lambda \operatorname{Id}_{3}$ for some $\lambda>0$, which occurs if and only if $h$ has an $\mathrm{A}_{4}$-stabilizer.

Note. This result classifies all Riemann curvature tensors associated with the metrics of Lagrangians in $\mathcal{S}^{6}$. Furthermore, it also performs this classification for the case of special Lagrangian 3 -folds in $\mathbb{C}^{3}$. This should help lead to a solution of the isometric embedding problem for these submanifolds.

Proof. Since the set of $A \in \mathrm{Sym}_{3}(\mathbb{R})$ with distinct eigenvalues is dense in $\mathrm{Sym}_{3}(\mathbb{R})$ and every element of $\operatorname{Sym}_{3}(\mathbb{R})$ is $\mathrm{SO}(3)$-equivalent to a diagonal matrix, we need only turn the strict inequalities in Proposition 5.3 to equalities to give our conditions.

Part (i) follows from (15). For (ii), notice that $\sigma(K)=0$ forces $\lambda_{1}=0$ and (16) leads to $\lambda_{2} \lambda_{3}=0$. The additional assumption that $K \neq 0$ means $\lambda_{2}=0$. Diagonalise $K$ to $\operatorname{diag}\left(\lambda_{3}, 0,0\right)$. We can explicitly calculate the cubics $h$ which map to this diagonal matrix as

$$
a y\left(y^{2}-3 z^{2}\right)+b z\left(3 y^{2}-z^{2}\right)
$$

where $a, b$ satisfy $2 a^{2}+2 b^{2}+\operatorname{Tr} K=0$. Using $\mathrm{SO}(3)$ to set $b=0$, we see from Table 1 that $h$ has an $\mathrm{S}_{3}$-stabilizer.

For (iii), by diagonalising $K=K(h)$ to $\operatorname{diag}\left(\lambda_{1}, \lambda_{2}, \lambda_{3}\right)$, we notice from the proof of Proposition 5.3 that we must have $h_{112}=h_{332}=h_{113}=h_{223}=h_{123}=0$. Therefore this $h$ is, up to sign,

$$
\left(\frac{p+q}{2}\right) x\left(2 x^{2}-3 y^{2}-3 z^{2}\right)+3\left(\frac{q-p}{2}\right) x\left(y^{2}-z^{2}\right),
$$

where $p=\sqrt{\frac{1}{2}\left(\lambda_{3}-\operatorname{Tr} K\right)}$ and $q=\sqrt{\frac{1}{2}\left(\lambda_{2}-\operatorname{Tr} K\right)}$. Notice that $p$ and $q$ are both non-zero, since otherwise $3 \sigma(K)=-\lambda_{1}^{2}<0$, a contradication. Moreover, $q^{2}-p^{2}=\frac{1}{2}\left(\lambda_{2}-\lambda_{3}\right)>0$. Thus, $h$ is a cubic with $\mathbb{Z}_{2}$-symmetry by Table 1 with parameters $r=\frac{1}{2}(p+q)$ and $s=\frac{1}{2}(q-p)$ satisfying $r>s>0$.

A cubic $h$ with $\mathbb{Z}_{2}$-symmetry, with parameters $r, s$ as in Table1, defines a matrix $K(h)$ with eigenvalues $r^{2}-s^{2}$ and $-3 r^{2}-s^{2} \pm 4 r s$. Thus, $K(h)$ has $\sigma(K)=\left(r^{2}-s^{2}\right)^{2}$. If the eigenvalues of $K(h)$ are $\lambda_{1}>\lambda_{2}>\lambda_{3}$, then $\lambda_{1}=r^{2}-s^{2}$ if $r>s$ and $\lambda_{2}=r^{2}-s^{2}$ if $r<s$. This proves (iii).

Now suppose there are at least two repeated eigenvalues for $K$ with $\lambda_{1}^{2}=\sigma(K)>$ 0 . If $\lambda_{1}>\lambda_{2}=\lambda_{3}, \lambda_{1}+2 \lambda_{2}=\operatorname{Tr} K$ and $3 \lambda_{1}^{2}=\lambda_{2}\left(2 \lambda_{1}+\lambda_{2}\right)>0$. We quickly see that $\lambda_{1}=-\frac{1}{5} \operatorname{Tr} K$ and $\lambda_{2}=\lambda_{3}=\frac{3}{5} \operatorname{Tr} K$. Again by diagonalising $K$ we can solve for $h$ as

$$
r x\left(2 x^{2}-3 y^{2}-3 z^{2}\right),
$$

where $5 r^{2}+\operatorname{Tr} K=0$. This $h$ has an $\mathrm{SO}(2)$-symmetry by Table 1 . If $\lambda_{1}=\lambda_{2}>\lambda_{3}$, the formulae $2 \lambda_{1}+\lambda_{3}=\operatorname{Tr} K, 3 \lambda_{1}^{2}=\lambda_{1}\left(2 \lambda_{3}+\lambda_{1}\right)$ and $\operatorname{Tr} K<0$ imply that $\lambda_{1}=\lambda_{2}=0$, but this has $\sigma(K)=0$, a contradiction. Part (iv) follows. 
Finally, suppose $K=-3 p^{2} I$ for $p>0$, which clearly has $\lambda_{1}^{2}=\sigma(K)>0$. Clearly, $h=3 \sqrt{3} p x\left(y^{2}-z^{2}\right)$ maps to $K$ and has an $\mathrm{A}_{4}$-stabilizer. However, we can explicitly calculate that the fibre of (14) at $K$ contains the aforementioned cubic together with cubics of the form

$$
p x\left(2 x^{2}-3 y^{2}-3 z^{2}\right)+\sqrt{2 p^{2}-q^{2}} y\left(y^{2}-3 z^{2}\right)+q z\left(3 y^{2}-z^{2}\right)
$$

for $q$ satisfying $q^{2} \leq 2 p^{2}$. Since we can use an $\mathrm{SO}(3)$ transformation to set $q=0$, the remarks before Table 1 show that these cubics also have an $\mathrm{A}_{4}$-stabilizer.

Remark. By the proof of Proposition 5.4(iii), $K(h)$ has eigenvalues $\lambda_{1}>\lambda_{2}>\lambda_{3}$ satisfying either $\lambda_{1}^{2}=\sigma(K)$ or $\lambda_{2}^{2}=\sigma(K)$ if and only if $h$ has a $\mathbb{Z}_{2}$-stabilizer.

Proposition 5.5. Let $K \in \operatorname{Sym}_{3}(\mathbb{R})$ and use the notation of Proposition 5.4. Suppose that $\lambda_{1}^{2}<\sigma(K), \operatorname{Tr} K<0$ and that $K=K(h)$ as in (14). Then $K$ has exactly two repeated eigenvalues if and only if $h$ has a $\mathbb{Z}_{3}$-stabilizer in $\mathrm{SO}(3)$.

Proof. Suppose first that the eigenvalues satisfy $\lambda_{1}>\lambda_{2}=\lambda_{3}$. By assumption, $3 \lambda_{1}^{2}<\lambda_{2}\left(2 \lambda_{1}+\lambda_{2}\right)$. Using $\lambda_{1}+2 \lambda_{2}=\operatorname{Tr} K$, we see that $\frac{3}{5} \operatorname{Tr} K<\lambda_{2}<\frac{1}{3} \operatorname{Tr} K$. We can write $\lambda_{2}=\lambda_{3}=-3 r^{2}$ for some $r>0$, since $\lambda_{2}<0$. We know that $\operatorname{Tr} K<-5 r^{2}$, so there exists $a>0$ such that $\operatorname{Tr} K=-5 r^{2}-2 a^{2}$. One quickly sees that $\lambda_{1}=r^{2}-2 a^{2}$ and that $\lambda_{2}<\frac{1}{3} \operatorname{Tr} K$ if and only if $a<r \sqrt{2}$. Diagonalising $K$, we recognise it as the image of cubics of the form

$$
r x\left(2 x^{2}-3 y^{2}-3 z^{2}\right)+\sqrt{a^{2}-b^{2}} y\left(y^{2}-3 z^{2}\right)+b z\left(3 y^{2}-z^{2}\right),
$$

where $b^{2} \leq a^{2}<2 r^{2}$. Using $\mathrm{SO}(3)$ to set $b=0$, we see from Table 1 that these cubics have a $\mathbb{Z}_{3}$-stabilizer. If $\lambda_{1}=\lambda_{2}>\lambda_{3}$, similar arguments show that $K$ is the image of cubics of the form (17), but now with $a>r \sqrt{2}$.

As a neat corollary, by analysing the proofs of Propositions 5.3 [5.5, we can collect some of our results concerning the Gauss map in terms of stabilizers of the fundamental cubic in $\mathrm{SO}(3)$.

Corollary 5.6. Let $K \in \operatorname{Sym}_{3}(\mathbb{R})$ with eigenvalues $\lambda_{1} \geq \lambda_{2} \geq \lambda_{3}$ be such that $K=K(h)$ as in (14). Use the notation of Proposition 5.4. We can present Table 2 for stabilizers of $h$ in $\mathrm{SO}(3)$, properties of $K$ and the dimension of the fibres of the Gauss map (14) at $K$.

TABLE 2. Fibres of the Gauss map and symmetries of the fundamental cubic

\begin{tabular}{|c|c|c|}
\hline Stabilizer of $h$ & Property of $K$ & Dimension \\
\hline $\mathrm{SO}(3)$ & $K=0$ & 0 \\
$\mathrm{SO}(2)$ & $\lambda_{1}^{2}=\sigma(K)>0$, so $\lambda_{1}>\lambda_{2}=\lambda_{3}$ & 2 \\
$\mathrm{~A}_{4}$ & $K=-\lambda \mathrm{Id}_{3}, \lambda>0$ & 3 \\
$\mathrm{~S}_{3}$ & $\lambda_{1}^{2}=\sigma(K)=0$, so $\lambda_{1}=\lambda_{2}=0>\lambda_{3}$ & 2 \\
$\mathbb{Z}_{3}$ & $\lambda_{1}^{2}<\sigma(K)$, two eigenvalues are repeated & 1 \\
$\mathbb{Z}_{2}$ & $\lambda_{1}>\lambda_{2}>\lambda_{3}, \lambda_{1}^{2}=\sigma(K)$ or $\lambda_{2}^{2}=\sigma(K)$ & 2 \\
0 & $\lambda_{1}>\lambda_{2}>\lambda_{3}, \lambda_{1}^{2}<\sigma(K)$ and $\lambda_{2}^{2} \neq \sigma(K)$ & 1 \\
\hline
\end{tabular}




\section{LAGRANGIAN SUBMANIFOLDS AND FUNDAMENTAL CUBICS WITH SYMMETRIES}

In this section we classify the families of Lagrangians in $\mathcal{S}^{6}$ whose fundamental cubic admits a pointwise symmetry. This is mainly a detailed survey of results by other authors, though we also include new results and observations.

For this section let $L$ be a Lagrangian submanifold of $\mathcal{S}^{6}$ with fundamental cubic $C_{L}$ and suppose, for simplicity, that it is connected. We use the notation of 3.1 . In particular, recall that $\left\{2 \omega_{1}, 2 \omega_{2}, 2 \omega_{3}\right\}$ defines an orthonormal coframe for $L$ and that $\alpha+[\omega]$ is the connection 1-form of the Levi-Civita connection of the metric $g_{L}$ on $L$. Since $\alpha$ is skew-symmetric, for convenience we shall write

$$
\alpha_{1}=\alpha_{23}, \quad \alpha_{2}=\alpha_{31}, \quad \alpha_{3}=\alpha_{12} .
$$

We organise our results and examples by the possible pointwise stabilizers of $C_{L}$ as given in Table 1. To rule out trivial cases we make the following definition.

Definition 6.1. A Lagrangian $L$ in $\mathcal{S}^{6}$ is simple if it is a totally geodesic $\mathcal{S}^{3}$.

6.1. $\mathrm{SO}(3)$. The stabilizer of $C_{L}$ in $\mathrm{SO}(3)$ is all of $\mathrm{SO}(3)$ at every point if and only if $C_{L}=0$ by Proposition 5.1. Using (7a) we see that

$$
\mathrm{d}(\alpha+[\omega])+(\alpha+[\omega]) \wedge(\alpha+[\omega])=4 \omega \wedge \omega^{\mathrm{T}}
$$

so $L$ has constant curvature 1 . Thus, $L$ is totally geodesic and hence simple.

Proposition 6.2. A connected Lagrangian submanifold of $\mathcal{S}^{6}$ whose fundamental cubic has an $\mathrm{SO}(3)$-stabilizer at each point is simple.

Example 6.3 (Simple case). A simple Lagrangian is the intersection of a linear coassociative 4-plane in $\operatorname{Im} \mathbb{O}$ with $\mathcal{S}^{6}$ by Proposition 2.5. Since $\mathrm{G}_{2}$ acts transitively on the set of coassociative 4-planes with isotropy $\mathrm{SO}(4)$, a simple Lagrangian has $\mathrm{SO}(4)$-symmetry and is (up to $\mathrm{G}_{2}$ transformation)

$$
L_{0}=\left\{y_{1} \varepsilon_{1}+y_{3} \varepsilon_{3}+y_{5} \varepsilon_{5}+y_{7} \varepsilon_{7}: y_{1}^{2}+y_{3}^{2}+y_{5}^{2}+y_{7}^{2}=1\right\},
$$

recalling the basis $\varepsilon_{i}$ for $\operatorname{Im} \mathbb{O}$. Notice that $L_{0}$ is trivially ruled. Furthermore, $L_{0}$ is a tube radius $\frac{\pi}{2}$ about a totally geodesic 2 -sphere, where the tube is defined using the standard Hopf fibration $\mathcal{S}^{3} \rightarrow \mathcal{S}^{2}$.

6.2. $\mathbf{S O}(\mathbf{2})$. To give a feel for later, more complicated, calculations which will often be suppressed, we go through this case in some detail.

If $C_{L} \neq 0$ has an $\mathrm{SO}(2)$-stabilizer at each point, then, by Proposition 5.1, there exist an open dense subset $L^{*}$ of $L$ and some function $r: L^{*} \rightarrow \mathbb{R}^{+}$such that $C_{L}=$ $r \omega_{1}\left(2 \omega_{1}^{2}-3 \omega_{2}^{2}-3 \omega_{3}^{2}\right)$ defines an $\mathrm{SO}(2)$-subbundle $\mathcal{F}$ of the adapted frame bundle over $L^{*}$. Since $\mathcal{F}$ is an $\mathrm{SO}(2)$-bundle there exist functions $t_{i j}$ for $i=2,3, j=1,2,3$ such that $\alpha_{2}=t_{2 j} \omega_{j}$ and $\alpha_{3}=t_{3 j} \omega_{j}$, using summation notation. Moreover, there exist functions $r_{i}$ for $i=1,2,3$ such that $\mathrm{d} r=r_{i} \omega_{i}$.

Define $\beta_{i j}$ in terms of $r$ and $\omega_{i}$ using (6b). The equations (6a) and (6d) then give $t_{21}=t_{31}=r_{2}=r_{3}=0, t_{22}=t_{33}=-\frac{1}{2}, t_{23}=-t_{32}=t$ and $r_{1}=-4 r t$. Putting this information in (6c) forces $t=0$ and $r=2 \sqrt{5}$. Thus, we may take $L^{*}=L$ and see that $C_{L}=2 \sqrt{5} \omega_{1}\left(2 \omega_{1}^{2}-3 \omega_{2}^{2}-3 \omega_{3}^{2}\right)$ over $L$. 
The second structure equations we have so far are

$$
\begin{gathered}
\mathrm{d} \omega_{1}=\omega_{2} \wedge \omega_{3} ; \quad \mathrm{d} \omega_{2}=\omega_{3} \wedge\left(\alpha_{1}+\frac{3}{2} \omega_{1}\right) ; \quad \mathrm{d} \omega_{3}=\left(\alpha_{1}+\frac{3}{2} \omega_{1}\right) \wedge \omega_{2} ; \\
\mathrm{d}\left(\alpha_{1}+\frac{3}{2} \omega_{1}\right)=6 \omega_{2} \wedge \omega_{3} .
\end{gathered}
$$

We see that the structure equations for $\omega_{2}$ and $\omega_{3}$ define a constant curvature 2-sphere. Equations (5) with $\omega_{2}=\omega_{3}=0$ give

$$
\mathrm{d} \mathbf{x}=2 \mathbf{e}_{1} \omega_{1} ; \quad \mathrm{d} \mathbf{e}_{1}=-2 \mathbf{x} \omega_{1}+\sqrt{5} J \mathbf{e}_{1} \omega_{1} ; \quad \mathrm{d} J \mathbf{e}_{1}=-\sqrt{5} \mathbf{e}_{1} \omega_{1} .
$$

Clearly, (19) defines a circle with radius $\frac{2}{3}$.

We also notice that the Lie derivative $\mathcal{L}_{\mathbf{e}_{1}}\left(\omega_{1}^{2}+\omega_{2}^{2}+\omega_{3}^{2}\right)=0$, so that $\mathbf{e}_{1}$ is a Killing vector for the metric. Thus, $L$ is homogeneous and topologically $\mathcal{S}^{3}$ fibred by circles over $\mathcal{S}^{2}$. Moreover, by inspection of (18), $L$ is an $\mathrm{SU}(2)$-orbit in $\mathcal{S}^{6}$ for some $\mathrm{SU}(2)$ subgroup $\mathrm{G}$ of $\mathrm{G}_{2}$. Clearly, $\mathrm{G}$ must have a commuting $\mathrm{U}(1)$ subgroup in $\mathrm{G}_{2}$ because of the circle fibration. Calculating the eigenvalues of the generators of the Lie algebra $\mathfrak{g}$ of $\mathrm{G}$, we recognise its action on $\operatorname{Im} \mathbb{O} \cong \mathbb{R}^{3} \oplus \mathbb{C}^{2}$ as $\mathrm{SU}(2)$ on $\mathbb{C}^{2}$ and $\mathrm{SO}(3)$ on $\mathbb{R}^{3}$. Here, $\mathbb{R}^{3}$ is spanned by $\left\{\varepsilon_{1}, \varepsilon_{2}, \varepsilon_{3}\right\}$ and $\mathbb{C}^{2}$ by $\left\{\varepsilon_{4}+i \varepsilon_{6}, \varepsilon_{5}+i \varepsilon_{7}\right\}$. Explicitly, the generators of $\mathfrak{g}$ are

$$
\begin{aligned}
& U_{1}=-2 E_{23}+E_{45}+E_{67} ; \\
& U_{2}=-2 E_{31}+E_{46}-E_{57} ; \\
& U_{3}=-2 E_{12}-E_{47}-E_{56},
\end{aligned}
$$

where

$$
E_{i j}\left(\varepsilon_{k}\right)= \begin{cases}\varepsilon_{i} & \text { if } j=k, \\ -\varepsilon_{j} & \text { if } k=i, \\ 0 & \text { otherwise. }\end{cases}
$$

Harvey and Lawson [14, Theorem IV.3.2] classify the coassociative submanifolds invariant under this $\mathrm{SU}(2)$ action, hence the Lagrangian submanifolds which are orbits of this action on $\mathcal{S}^{6}$. This result also follows from [22, Theorem 4.1].

Example 6.4 (A "squashed" 3-sphere). The 3-dimensional submanifold of $\mathcal{S}^{6}$ given by

$$
L_{1}=\left\{\frac{\sqrt{5}}{3} \bar{q} \varepsilon_{1} q+\frac{2}{3} q \varepsilon_{5}: q \in\left\langle 1, \varepsilon_{1}, \varepsilon_{2}, \varepsilon_{3}\right\rangle_{\mathbb{R}} \text { with }|q|=1\right\}
$$

is Lagrangian. Let $\mathcal{S}^{3}$ be the unit 3 -sphere in $\mathbb{R}^{4}$ with coordinates $x_{1}, x_{2}, x_{3}, x_{4}$. Following [7, Example 5.1], define vector fields on $\mathcal{S}^{3}$ by

$$
\begin{aligned}
& \mathbf{w}_{1}=x_{2} \mathbf{v}_{1}-x_{1} \mathbf{v}_{2}+x_{4} \mathbf{v}_{3}-x_{3} \mathbf{v}_{4} ; \\
& \mathbf{w}_{2}=x_{3} \mathbf{v}_{1}-x_{4} \mathbf{v}_{2}-x_{1} \mathbf{v}_{3}+x_{2} \mathbf{v}_{4} ; \\
& \mathbf{w}_{3}=x_{4} \mathbf{v}_{1}+x_{3} \mathbf{v}_{2}-x_{2} \mathbf{v}_{3}-x_{1} \mathbf{v}_{4},
\end{aligned}
$$

where $\mathbf{v}_{i}=\frac{\partial}{\partial x_{i}}$. These vectors form a basis for $T \mathcal{S}^{3}$, so we can define a metric $g_{1}$ on $\mathcal{S}^{3}$ by requiring that the $\mathbf{w}_{i}$ are orthogonal with respect to $g_{1}$,

$$
g_{1}\left(\mathbf{w}_{1}, \mathbf{w}_{1}\right)=\frac{4}{9} \quad \text { and } \quad g_{1}\left(\mathbf{w}_{2}, \mathbf{w}_{2}\right)=g_{1}\left(\mathbf{w}_{3}, \mathbf{w}_{3}\right)=\frac{8}{3} .
$$

By [7, Theorem 5.1], $L_{1}$ is the isometric embedding of $\left(\mathcal{S}^{3}, g_{1}\right)$ via the map $q \mapsto$ $\frac{\sqrt{5}}{3} \bar{q} \varepsilon_{1} q+\frac{2}{3} q \varepsilon_{5}$, where we identify $\mathcal{S}^{3}$ with the unit sphere in $\left\langle 1, \varepsilon_{1}, \varepsilon_{2}, \varepsilon_{3}\right\rangle_{\mathbb{R}}$. 
Remarks. By scaling $L_{1}$, we recognise it as the graph of the Hopf map $\mathcal{S}^{3} \rightarrow \mathcal{S}^{2}$ given by $q \mapsto \frac{\sqrt{5}}{2} \bar{q} \varepsilon_{1} q$. Furthermore, although $L_{1}$ is an $\mathrm{SU}(2)$-orbit, it is in fact invariant under an action of $\mathrm{U}(2)$, since there is an extra commuting $\mathrm{U}(1)$-action which results from the circle fibration.

Proposition 6.5. The unique (up to rigid motion) connected, non-simple Lagrangian submanifold of $\mathcal{S}^{6}$ whose fundamental cubic has an $\mathrm{SO}(2)$-stabilizer at each point is $L_{1}$ as given in Example 6.4.

From Definition 4.7 and Example 6.4 we see that $L_{1}$ is a tube of radius $\sin ^{-1}\left(\frac{2}{3}\right)$ about a totally geodesic 2 -sphere.

6.3. $\mathbf{A}_{4}$. If $C_{L} \neq 0$ has an $\mathrm{A}_{4}$-stabilizer at each point, then, by Proposition 5.1, there exist an open dense subset $L^{*}$ of $L$ and a function $s: L^{*} \rightarrow \mathbb{R}^{+}$, such that $C_{L}=3 s \omega_{1}\left(\omega_{2}^{2}-\omega_{3}^{2}\right)$ defines an $\mathrm{A}_{4}$-subbundle $\mathcal{F}$ of the adapted frame bundle over $L^{*}$. Therefore, there exist functions $t_{i j}$ on $\mathcal{F}$ such that $\alpha_{i}=t_{i j} \omega_{j}$. Using (6), we find that $\alpha=-\frac{1}{2}[\omega]$ and $s=2 \sqrt{15}$. Therefore, we can take $L^{*}=L$, and $C_{L}=12 \sqrt{15} \omega_{1}\left(\omega_{2}^{2}-\omega_{3}^{2}\right)$ over $L$. Equation (7a) gives

$$
\mathrm{d}(\alpha+[\omega])+(\alpha+[\omega]) \wedge(\alpha+[\omega])=\frac{1}{4} \omega \wedge \omega^{\mathrm{T}},
$$

so $L$ has constant curvature $\frac{1}{16}$. By Proposition [5.4(v), our calculations here and in $₫ 6.1$ prove [9, Theorem 2]: the only constant curvature Lagrangian submanifolds of $\mathcal{S}^{6}$ have curvature 1 or $\frac{1}{16}$.

Further, by [22, Lemma 2.5 \& Theorem 4.3(i)], $L$ must be, up to rigid motion, the orbit through $\varepsilon_{2}$ of the 3 -dimensional closed Lie subgroup $\mathrm{G}$ of $\mathrm{G}_{2}$ whose Lie algebra has the following generators:

$$
\begin{aligned}
& U_{1}=4 E_{32}+2 E_{54}+6 E_{76} ; \\
& U_{2}=\sqrt{6}\left(2 E_{51}-E_{62}+E_{73}\right)+\sqrt{10}\left(E_{42}+E_{53}\right) ; \\
& U_{3}=\sqrt{6}\left(2 E_{41}+E_{63}+E_{72}\right)+\sqrt{10}\left(E_{43}-E_{52}\right),
\end{aligned}
$$

where $E_{i j}$ is given by (20). We have used the fact that all constant curvature $\frac{1}{16}$ Lagrangians are congruent up to $\mathrm{G}_{2}$ transformation. The Lie group $\mathrm{G}$ is the $\mathrm{SO}(3)$ subgroup of $\mathrm{SO}(7)$ which acts irreducibly on $\mathbb{R}^{7} \cong \operatorname{Im} \mathbb{O}$. We can interpret the group action as follows.

Example 6.6 $(\mathrm{SO}(3)$-orbits 1$)$. Identify $\operatorname{Im} \mathbb{O}$ with the homogeneous harmonic cubics $\mathcal{H}^{3}\left(\mathbb{R}^{3}\right)$ on $\mathbb{R}^{3}$ by

$$
\begin{array}{rlrl}
\varepsilon_{1} & \mapsto \frac{\sqrt{10}}{10} x\left(2 x^{2}-3 y^{2}-3 z^{2}\right) ; & & \\
\varepsilon_{2} \mapsto-\sqrt{6} x y z ; & \varepsilon_{3} \mapsto \frac{\sqrt{6}}{2} x\left(y^{2}-z^{2}\right) ; \\
\varepsilon_{4} \mapsto-\frac{\sqrt{15}}{10} y\left(4 x^{2}-y^{2}-z^{2}\right) ; & \varepsilon_{5} \mapsto-\frac{\sqrt{15}}{10} z\left(4 x^{2}-y^{2}-z^{2}\right) ; \\
\varepsilon_{6} \mapsto \frac{1}{2} y\left(y^{2}-3 z^{2}\right) ; & \varepsilon_{7} \mapsto-\frac{1}{2} z\left(z^{2}-3 y^{2}\right) .
\end{array}
$$


Notice that the cubics above are of unit norm. We then recognise the generators (21) of the Lie algebra of $\mathrm{G}$ as acting as

$$
U_{1}=2\left(y \frac{\partial}{\partial z}-z \frac{\partial}{\partial y}\right), U_{2}=2\left(z \frac{\partial}{\partial x}-x \frac{\partial}{\partial z}\right), U_{3}=2\left(x \frac{\partial}{\partial y}-y \frac{\partial}{\partial x}\right) .
$$

Thus the action of $\mathrm{G}$ is simply the standard $\mathrm{SO}(3)$ action on $\mathcal{H}^{3}\left(\mathbb{R}^{3}\right)$, under this particular identification with $\operatorname{Im} \mathbb{O}$.

With this identification, the $\mathrm{SO}(3)$-orbit $L_{2}$ of $-\sqrt{6} x y z$ is Lagrangian in $\mathcal{S}^{6}$ and has constant curvature $\frac{1}{16}$. Moreover, $L_{2}$ is diffeomorphic to $\mathrm{SO}(3) / \mathrm{A}_{4}$ by Table 1. There is an explicit description of $L_{2}$ in [7, Example 2] as a 24-fold isometric immersion of $\mathcal{S}^{3}\left(\frac{1}{16}\right)$ in terms of harmonic polynomials of degree 6 .

Remark. The main result of [7] is that $L_{1}$ and $L_{2}$ given in Examples 6.4 and 6.6. respectively, together with the simple example $L_{0}$, classify all Lagrangians whose sectional curvatures are bounded below by $\frac{1}{16}$.

From our discussion, we can deduce the following result.

Proposition 6.7. The unique (up to rigid motion) connected, non-simple Lagrangian submanifold of $\mathcal{S}^{6}$ whose fundamental cubic has an $\mathrm{A}_{4}$-stabilizer at each point is $L_{2}$ as given in Example 6.6.

Remark. In contrast, the only special Lagrangian 3-folds in $\mathbb{C}^{3}$ whose fundamental cubic has a pointwise $\mathrm{A}_{4}$-symmetry are 3 -planes [3, Theorem 2].

To see the ruling of $L_{2}$, we first notice, by the remarks after Proposition [5.1, that $C_{L_{2}}$ is $\mathrm{SO}(3)$-equivalent to $2 \sqrt{5} \omega_{1}\left(2 \omega_{1}^{2}-3 \omega_{2}^{2}-3 \omega_{3}^{2}\right)+2 \sqrt{10} \omega_{2}\left(\omega_{2}^{2}-3 \omega_{3}^{2}\right)$. The second structure equations are

$$
\mathrm{d} \omega_{1}=\omega_{2} \wedge \omega_{3} ; \quad \mathrm{d} \omega_{2}=\omega_{3} \wedge \omega_{1} ; \quad \mathrm{d} \omega_{3}=\omega_{1} \wedge \omega_{2} .
$$

By inspection, the equations for $\omega_{2}$ and $\omega_{3}$ define a 2-sphere of constant curvature. Moreover, the first structure equations with $\omega_{2}=\omega_{3}=0$ yield equations (19) as in the $\mathrm{SO}(2)$-stabilizer case and hence define a circle of radius $\frac{2}{3}$. Thus $L_{2}$ has a $\frac{2}{3}$-ruling over a constant curvature $\mathcal{S}^{2}$.

This 2-sphere cannot be totally geodesic, otherwise the corresponding Lagrangian would be $L_{1}$ given in Example 6.4. By [23, Theorem B], the only possible constant Gauss curvatures of pseudoholomorphic curves in $\mathcal{S}^{6}$ are $0, \frac{1}{6}$ and 1 . Therefore the $\mathcal{S}^{2}$ must have constant curvature $\frac{1}{6}$ and, by [2, Theorem 4.6], have null-torsion. Thus, this 2-sphere is congruent up to $\mathrm{G}_{2}$ transformations to the Borůvka sphere $\mathcal{S}^{2}\left(\frac{1}{6}\right)$ in $\mathcal{S}^{6}$, which is the orbit of $\epsilon_{1}$, or equivalently $\frac{\sqrt{10}}{10} x\left(2 x^{2}-3 y^{2}-3 z^{2}\right)$, under the $\mathrm{SO}(3)$ action described in Example 6.6.

As noted in [10, p. 123], and as one could quickly verify using the structure equations, $L_{2}$ is a tube of radius $\sin ^{-1}\left(\frac{2}{3}\right)$ about $\mathcal{S}^{2}\left(\frac{1}{6}\right)$ in the second normal bundle. We shall see that constructing a quasi-ruled Lagrangian tube about a non-totally geodesic pseudoholomorphic curve in the second normal bundle is possible if and only if the curve has null-torsion and the radius is $\sin ^{-1}\left(\frac{2}{3}\right)$.

6.4. $\mathbf{S}_{3}$. Suppose $C_{L} \neq 0$ has a pointwise $\mathrm{S}_{3}$-stabilizer. Then there is an open dense subset $L^{*}$ of $L$ and a function $a: L^{*} \rightarrow \mathbb{R}^{+}$such that $C_{L}=a \omega_{2}\left(\omega_{2}^{2}-3 \omega_{3}^{2}\right)$ defines an $\mathrm{S}_{3}$-subbundle $\mathcal{F}$ of the adapted frame bundle over $L^{*}$.

By Proposition 5.4(ii), the symmetric matrix $K$ associated with the Riemann curvature tensor of $L$, as defined in Definition 5.2, has a repeated eigenvalue of 0 . 
Thus, by the remark following Definition 5.2 $L$ is quasi-Einstein; that is, its Ricci tensor has repeated eigenvalues. Quasi-Einstein Lagrangians in $\mathcal{S}^{6}$ are classified in [6] - more on this later. We show that the Lagrangians whose fundamental cubic has a pointwise $\mathrm{S}_{3}$-stabilizer at every point are in correspondence with the non-simple Lagrangians satisfying Chen's equality.

Definition 6.8. Chen [4 introduced a new Riemannian invariant $\delta_{M}$ to study submanifolds $M^{n}$ of spaces of constant curvature. Explicitly, if $s$ is the sectional curvature of $M, p \in M,\left\{\mathbf{v}_{1}, \ldots, \mathbf{v}_{n}\right\}$ is an orthonormal basis for $T_{p} M$ and $\mathcal{P}$ is the set of 2-planes in $T_{p} M$,

$$
\delta_{M}(p)=\sum_{i<j} s\left(\mathbf{v}_{i} \wedge \mathbf{v}_{j}\right)-\inf _{\Pi \in \mathcal{P}} s(\Pi)
$$

When $M^{n}$ is a minimal submanifold of a manifold with constant curvature $c$, it follows from [4, Lemma 3.2] that $\delta_{M} \leq \frac{1}{2}(n+1)(n-2) c$. Thus, Chen's equality, which is for minimal 3 -dimensional submanfolds of $\mathcal{S}^{6}$, is $\delta_{M}=2$.

Lemma 6.9. A non-simple Lagrangian in $\mathcal{S}^{6}$ has a fundamental cubic with an $\mathrm{S}_{3}$-stabilizer at each point if and only if it satisfies Chen's equality.

Proof. By [8, Lemma 3.1], $L$ is non-simple and satisfies Chen's equality if and only if there exists a non-zero tangent vector $\mathbf{t}$ on $L$ such that $h_{L}(\mathbf{t}, \mathbf{v})=0$ for all tangent vectors $\mathbf{v}$ on $L$. Letting $\mathbf{t}=\mathbf{e}_{1}$, we see that the fundamental cubic of $L$ satisfying Chen's equality must be of the form

$$
a \omega_{2}\left(\omega_{2}^{2}-3 \omega_{3}^{2}\right)+b \omega_{3}\left(3 \omega_{2}^{2}-\omega_{3}^{2}\right)
$$

Since we need only fix $\mathbf{e}_{1}$ in our frame, we can set $b=0$ by using $\mathrm{SO}(2)$.

We shall see that the Lagrangians whose fundamental cubic has pointwise $\mathrm{S}_{3^{-}}$ symmetry include those associated with lower-dimensional geometries. This leads us to prove the following result.

Proposition 6.10. Identify $\operatorname{Im} \mathbb{O} \cong \mathbb{R} \oplus \mathbb{C}^{3}$ such that if $\left(x_{1}, \ldots, x_{7}\right)$ are coordinates on $\operatorname{Im} \mathbb{O}$, the coordinates on $\mathbb{R}$ and $\mathbb{C}^{3}$ are $x_{1}$ and $\left(x_{2}+i x_{3}, x_{4}+i x_{5}, x_{6}+i x_{7}\right)$, respectively. Recall that $\mathcal{S}^{5} \subseteq \mathbb{C}^{3}$ is endowed with a contact structure.

(i) $L=\{0\} \times P \subseteq\{0\} \times \mathcal{S}^{5} \subseteq \mathcal{S}^{6}$ is Lagrangian if and only if $P$ is the link in $\mathcal{S}^{5}$ of a complex 2 -dimensional cone in $\mathbb{C}^{3}$.

(ii) $L=\left\{(\cos t, p \sin t) \in \mathbb{R} \oplus \mathbb{C}^{3}: p \in \Sigma \subseteq \mathcal{S}^{5}, t \in(0, \pi)\right\} \subseteq \mathcal{S}^{6}$ is Lagrangian if and only if $\Sigma$ is a minimal Legendrian surface in $\mathcal{S}^{5}$.

Proof. Use the notation of Definition 2.2 and use $\omega_{0}$ and $\Omega_{0}$ to denote the Kähler and holomorphic volume forms on $\mathbb{C}^{3}$. We can write $\varphi_{0}$ on $\operatorname{Im} \mathbb{O} \cong \mathbb{R} \oplus \mathbb{C}^{3}$ as

$$
\varphi_{0}=\mathrm{d} x_{1} \wedge \omega_{0}+\operatorname{Re} \Omega_{0} .
$$

By Proposition 2.5, $L$ is Lagrangian in $\mathcal{S}^{6}$ if and only if the cone $N$ on $L$ is coassociative; that is, satisfies $\left.\varphi_{0}\right|_{N} \equiv 0$. By (22), $N=\{0\} \times X$ is coassociative in $\operatorname{Im} \mathbb{O} \cong \mathbb{R} \oplus \mathbb{C}^{3}$ if and only if $X$ is a complex surface. Part (i) follows. Similarly, $N=\mathbb{R} \times Y$ is coassociative if and only if $Y$ is special Lagrangian with phase $-i$ in $\mathbb{C}^{3}$ by Definition 2.7. Since the link of a special Lagrangian in $\mathbb{C}^{3}$ is minimal Legendrian in $\mathcal{S}^{5}$, part (ii) is also proved.

Lagrangian submanifolds in $\mathcal{S}^{6}$ satisfying Chen's equality are classified in [8]. We review these results below. 
Example 6.11 (Links of complex cones). Let $\mathbf{u}: \Sigma \rightarrow \mathbb{C P}^{2}$ be a holomorphic curve. Let $\mathcal{C}(\Sigma)$ be the circle bundle over $\Sigma$ induced by the Hopf fibration $\mathcal{S}^{5} \rightarrow \mathbb{C P}^{2}$. Let $\mathrm{x}: \mathcal{C}(\Sigma) \rightarrow \mathcal{S}^{5}$ be such that the following diagram commutes:

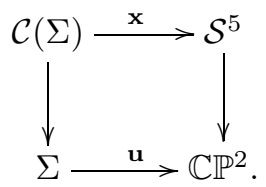

By [8, Theorem 1], there exists a totally geodesic embedding $i: \mathcal{S}^{5} \rightarrow \mathcal{S}^{6}$ such that $i \circ \mathbf{x}: \mathcal{C}(\Sigma) \rightarrow \mathcal{S}^{6}$ is a Lagrangian immersion satisfying Chen's equality. Let $L_{3}(\mathbf{u}, \Sigma)=i \circ \mathbf{x}(\mathcal{C}(\Sigma))$.

By Proposition 6.10, these examples are the links of complex cones embedded in a totally geodesic 5 -sphere in $\mathcal{S}^{6}$. Moreover, they are clearly tubes of radius $\frac{\pi}{2}$, in the plane bundle defined by the Hopf fibration, about the surface which is the embedding of $\Sigma$ in $\mathcal{S}^{6}$.

Note. The Lagrangian $L_{3}(\mathbf{u}, \Sigma)$ where $\Sigma$ is a totally geodesic $\mathbb{C P}^{1}$ is simple.

Example 6.12 (Ruled tubes in the second normal bundle). Let $\mathbf{u}: \Sigma \rightarrow \mathcal{S}^{6}$ be a non-totally geodesic pseudoholomorphic curve, let $h_{\Sigma}$ be its second fundamental form and let $\mathcal{U}(\Sigma)$ be its unit tangent bundle. If $\Sigma$ has no totally geodesic points or branch points, we can define a map $\mathrm{x}: \mathcal{U}(\Sigma) \rightarrow \mathcal{S}^{6}$ by

$$
\mathbf{x}: \mathbf{t} \mapsto \mathbf{t} \times \frac{h_{\Sigma}(\mathbf{t}, \mathbf{t})}{\left\|h_{\Sigma}(\mathbf{t}, \mathbf{t})\right\|} .
$$

By [8, Theorem 2], $\mathbf{x}$ defines a (possibly branched) Lagrangian immersion satisfying Chen's equality.

From (9) and Definition 4.7, we recognise $\mathbf{x}$ as defining a tube of radius $\frac{\pi}{2}$ in $N_{2} \Sigma$ about $\Sigma$. Note that we can extend the definition of $\mathbf{x}$ if $\Sigma$ has isolated branch and totally geodesic points, and still get a Lagrangian immersion by 8 , Theorem 3]. Let $L_{4}(\mathbf{u}, \Sigma)$ be the Lagrangian associated with $\mathbf{u}: \Sigma \rightarrow \mathcal{S}^{6}$.

From $[8$, Theorems $4 \& 5]$ and Lemma $[6.9$, we deduce the following.

Proposition 6.13. Let $L$ be a connected, non-simple Lagrangian in $\mathcal{S}^{6}$ with a fundamental cubic $C_{L}$.

(i) If $L$ is not linearly full in $\mathcal{S}^{6}, C_{L}$ has a pointwise $\mathrm{S}_{3}$-stabilizer and there exists a non-totally geodesic holomorphic curve $\mathbf{u}: \Sigma \rightarrow \mathbb{C P}^{2}$ such that $L=L_{3}(\mathbf{u}, \Sigma)$, as given in Example 6.11.

(ii) Suppose $L$ is linearly full and $C_{L}$ has a pointwise $\mathrm{S}_{3}$-stabilizer. There is an open dense subset $L^{*}$ of $L$ such that, for all $x \in L^{*}$, there exist an open set $U \ni x$ and a non-totally geodesic pseudoholomorphic curve $\mathbf{u}: \Sigma \rightarrow \mathcal{S}^{6}$ such that $U \cap L^{*}=L_{4}(\mathbf{u}, \Sigma)$ as given by Example 6.12 .

Notice that the Lagrangians in Examples 6.11 and 6.12 are both ruled, as they are tubes of radius $\frac{\pi}{2}$. Therefore, the Lagrangians in $\mathcal{S}^{6}$ whose fundamental cubic has an $\mathrm{S}_{3}$-symmetry at each point are ruled.

For comparison later, as well as for interest, we derive the structure equations for Lagrangians with pointwise $\mathrm{S}_{3}$-symmetry of their fundamental cubic. 
Recall that we are working on an $\mathrm{S}_{3}$-bundle $\mathcal{F}$ over the open dense subset $L^{*}$ of $L$, so there exist functions $t_{i j}$ such that $\alpha_{i}=t_{i j} \omega_{j}$. Using (6a), (6b) and (6d), we have that $t_{22}=t_{33}=-2-3 t_{11}, t_{23}=-t_{32}$ and $t_{21}=t_{31}=0$. If we let $t_{1}=t_{23}$, $t_{2}=-t_{13}, t_{3}=t_{12}$ and $t_{0}=t_{11}$, then

$$
\begin{aligned}
& \alpha_{1}=t_{0} \omega_{1}+t_{3} \omega_{2}-t_{2} \omega_{3} ; \\
& \alpha_{2}=-\left(2+3 t_{0}\right) \omega_{2}+t_{1} \omega_{3} ; \\
& \alpha_{3}=-t_{1} \omega_{2}-\left(2+3 t_{0}\right) \omega_{3} ; \\
& \mathrm{d} a=-a\left(t_{1} \omega_{1}+3 t_{2} \omega_{2}+3 t_{3} \omega_{3}\right) .
\end{aligned}
$$

It follows that

$$
\begin{aligned}
& \mathrm{d} \omega_{1}=-2\left(1+3 t_{0}\right) \omega_{2} \wedge \omega_{3} \\
& \mathrm{~d} \omega_{2}=-t_{3} \omega_{2} \wedge \omega_{3}-2 t_{0} \omega_{3} \wedge \omega_{1}+t_{1} \omega_{1} \wedge \omega_{2} \\
& \mathrm{~d} \omega_{3}=t_{2} \omega_{2} \wedge \omega_{3}-t_{1} \omega_{3} \wedge \omega_{1}-2 t_{0} \omega_{1} \wedge \omega_{2} .
\end{aligned}
$$

Moreover, (6c) and $\mathrm{d}(\mathrm{d} a)=0$ imply that there exist $u_{1}, u_{2}, v_{1}, v_{2}$ such that

$$
\begin{aligned}
\mathrm{d} t_{0}=- & \frac{2}{3}\left(1+3 t_{0}\right) t_{1} \omega_{1}+u_{1} \omega_{2}+u_{2} \omega_{3} ; \\
\mathrm{d} t_{1}=( & \left.t_{0}^{2}+6 t_{0}-3-t_{1}^{2}\right) \omega_{1}-3 u_{2} \omega_{2}+3 u_{1} \omega_{3} ; \\
\mathrm{d} t_{2}=( & \left.t_{0} t_{3}-t_{1} t_{2}-u_{2}\right) \omega_{1} \\
& +\left(\frac{1}{2}\left(\frac{1}{8} a^{2}-t_{1}^{2}-t_{2}^{2}-t_{3}^{2}-7-14 t_{0}-15 t_{0}^{2}\right)+v_{1}\right) \omega_{2} \\
& +\left(v_{2}-\frac{1}{3}\left(1+3 t_{0}\right) t_{1}\right) \omega_{3} ; \\
\mathrm{d} t_{3}=( & \left.u_{1}-2 t_{0} t_{2}-t_{3} t_{1}\right) \omega_{1}+\left(v_{2}+\frac{1}{3}\left(1+3 t_{0}\right) t_{1}\right) \omega \\
& +\left(\frac{1}{2}\left(\frac{1}{8} a^{2}-t_{1}^{2}-t_{2}^{2}-t_{3}^{2}-7-14 t_{0}-15 t_{0}^{2}\right)-v_{1}\right) \omega_{3} .
\end{aligned}
$$

The appropriate EDS associated with these equations is involutive with the last non-zero character $s_{1}=4$. This is in agreement with Proposition 6.13, since this is the same local dependence as a pseudoholomorphic curve in $\mathcal{S}^{6}$.

To consider some important reductions of this system and for comparison later, we study these structure equations more thoroughly. Recall the observations in $\$ 3.2$ and define

$$
\begin{gathered}
\mathbf{u}=-J \mathbf{e}_{1} ; \\
\mathbf{f}_{1}=\frac{1}{2}\left(-J \mathbf{e}_{2}+i J \mathbf{e}_{3}\right) ; \quad \mathbf{f}_{2}=\frac{1}{2}\left(\mathbf{e}_{2}+i \mathbf{e}_{3}\right) ; \quad \mathbf{f}_{3}=\frac{1}{2}\left(\mathbf{x}+i \mathbf{e}_{1}\right) ; \\
\theta_{1}=\frac{1}{2}\left(3\left(1+t_{0}\right)+i t_{1}\right)\left(\omega_{2}+i \omega_{3}\right) ; \\
\kappa_{22}=i\left(\left(1+t_{0}\right) \omega_{1}+t_{3} \omega_{2}-t_{2} \omega_{3}\right) ; \quad \kappa_{33}=-2 i \omega_{1} ; \\
\kappa_{21}=k_{2} \theta_{1}=\frac{a}{4}\left(\omega_{2}+i \omega_{3}\right) ; \quad \kappa_{31}=k_{3} \theta_{1}=0 \\
\kappa_{32}=k_{1} \theta_{1}=\frac{1}{2}\left(\left(3 t_{0}-1\right)+i t_{1}\right)\left(\omega_{2}+i \omega_{3}\right) .
\end{gathered}
$$


These functions and forms satisfy the structure equations (11) for the adapted frame bundle of a pseudoholomorphic curve in $\mathcal{S}^{6}$. Notice from (111) and (26) that $J \mathbf{e}_{1}$ is constant if and only if $\theta_{1}=0$, which occurs if and only if $1+t_{0}=t_{1}=0$. Thus, the Lagrangian lies in a totally geodesic $\mathcal{S}^{5}$ if and only if $\left(t_{0}, t_{1}\right)=(-1,0)$.

If $\left(t_{0}, t_{1}\right) \neq(-1,0), \theta_{1}$ is nowhere vanishing on some open dense set, and so $\mathbf{u}$ in (26a) defines a pseudoholomorphic curve $\Sigma$ in $\mathcal{S}^{6}$. Moreover, as $\kappa_{31}=0$ on $\Sigma$, the unitary frame $\left\{\mathbf{f}_{1}, \mathbf{f}_{2}, \mathbf{f}_{3}\right\}$ over $\Sigma$ is adapted so that $\mathbf{f}_{2}$ spans $N_{1} \Sigma$ and $\mathbf{f}_{3}$ spans $N_{2} \Sigma$. Since $\mathbf{x}=\operatorname{Re} \mathbf{f}_{3}$ and $\mathbf{e}_{1}=\operatorname{Im} \mathbf{f}_{3}$, the Lagrangian defined by $\mathbf{x}$ is a tube of radius $\frac{\pi}{2}$ in $N_{2} \Sigma$ about $\Sigma$ as claimed in Proposition 6.13(ii).

Suppose that $t_{1}=0$. Then either $t_{0}=-1$ or $t_{0}=\frac{1}{3}$, and in each case the reduced EDS is involutive with $s_{1}=2$. As noted above, the system for $\left(t_{0}, t_{1}\right)=$ $(-1,0)$ describes the Lagrangians given in Example 6.11. For $\left(t_{0}, t_{1}\right)=\left(\frac{1}{3}, 0\right)$, the Lagrangians must necessarily be locally of the form in Example 6.12 by Proposition 6.13. From (26),$\left(t_{0}, t_{1}\right)=\left(\frac{1}{3}, 0\right)$ if and only if the torsion $k_{1}=0$, so the Lagrangians are tubes about null-torsion pseudoholomorphic curves.

Suppose we consider the reduced system where $a$ is constant. This forces $t_{i}=0$ for $i=1,2,3$ and either $\left(a, t_{0}\right)=(8,-1)$ or $\left(a, t_{0}\right)=\left(\frac{8}{3} \sqrt{15}, \frac{1}{3}\right)$.

Example 6.14 $(\mathrm{SO}(3)$-orbits 2$)$. If $\left(a, t_{0}\right)=(8,-1)$, our comments above show that the Lagrangian lies in a totally geodesic $\mathcal{S}^{5}$ and that

$$
\mathrm{d} \omega_{1}=4 \omega_{2} \wedge \omega_{3} ; \quad \mathrm{d} \omega_{2}=2 \omega_{3} \wedge \omega_{1} ; \quad \mathrm{d} \omega_{3}=2 \omega_{1} \wedge \omega_{2} .
$$

We observe that the equations for $\omega_{2}$ and $\omega_{3}$ define a constant curvature 2-sphere which cannot be totally geodesic; otherwise the Lagrangian would be simple. Thus, the underlying holomorphic curve is the constant curvature degree $2 \mathbb{C P}^{1}$ in $\mathbb{C P}^{2}$. Further, it is a homogeneous submanifold of $\mathcal{S}^{6}$, so we deduce from 22, Theorems $4.2 \& 4.4]$ that it is invariant under an $\mathrm{SO}(3)$ action on $\operatorname{Im} \mathbb{O} \cong \mathbb{R} \oplus \mathbb{C}^{3}$, where $\mathrm{SO}(3)$ acts trivially on $\mathbb{R}$ and as the standard (real) $\mathrm{SO}(3)$ action on $\mathbb{C}^{3}$. Hence, up to rigid motion, the Lagrangian is

$$
\left\{\left(0, z_{1}, z_{2}, z_{3}\right) \in \mathbb{R} \oplus \mathbb{C}^{3}: z_{1}^{2}+z_{2}^{2}+z_{3}^{2}=0\right\} \cap \mathcal{S}^{6},
$$

which is the Hopf lift of the Veronese curve $\mathbf{u}: \mathbb{C P}^{1}(2) \rightarrow \mathbb{C P}^{2}$.

Example 6.15 (SO (3)-orbits 3). If $\left(a, t_{0}\right)=\left(\frac{8}{3} \sqrt{15}, \frac{1}{3}\right)$,

$$
\mathrm{d} \omega_{1}=-4 \omega_{2} \wedge \omega_{3} ; \quad \mathrm{d} \omega_{2}=-\frac{2}{3} \omega_{3} \wedge \omega_{1} ; \quad \mathrm{d} \omega_{3}=-\frac{2}{3} \omega_{1} \wedge \omega_{2}
$$

Again this is a homogeneous submanifold of $\mathcal{S}^{6}$, and so, by process of elimination, we can deduce from [22, Theorems $4.3 \& 4.4$ ] that it is (up to $\mathrm{G}_{2}$ transformation) the orbit through $\varepsilon_{6} \in \operatorname{Im} \mathbb{O}$ of the $\mathrm{SO}(3)$ action given in Example 6.6. Equivalently, it is the $\mathrm{SO}(3)$-orbit of the cubic $\frac{1}{2} y\left(y^{2}-3 z^{2}\right)$ in $\mathcal{H}^{3}\left(\mathbb{R}^{3}\right)$, which is clearly diffeomorphic to $\mathrm{SO}(3) / \mathrm{S}_{3}$ by Table 1 . Moreover, the structure equations for $\omega_{2}$ and $\omega_{3}$ define a constant curvature 2-sphere, and, since the Lagrangian is linearly full, it must once again be the Borůva sphere. Hence this example is a tube of radius $\frac{\pi}{2}$ in the second normal bundle about $\mathcal{S}^{2}\left(\frac{1}{6}\right)$.

The author, in 21], gave a method for producing examples of coassociative 4folds in $\operatorname{Im} \mathbb{O}$ with symmetries. We can apply this method to the $\mathrm{SO}(3)$-action given in Example 6.6, and conical solutions will give rise to the Lagrangian $\mathrm{SO}(3)-$ orbits by Proposition 2.5. Altogether, we can derive a system of first-order ordinary 
differential equations whose solutions define the homogeneous Lagrangians we are interested in. Therefore, in principle, this system can simply be integrated to produce an explicit description of the Lagrangian given in Example 6.15, though the author has been unable to do this.

Note. Examples 6.3, 6.4, 6.6, 6.14 and 6.15 classify the homogeneous Lagrangian submanifolds of $\mathcal{S}^{6}$ up to $\mathrm{G}_{2}$ transformation, as studied in [22].

Example 6.16 (Products 1). Setting $t_{0}=-\frac{1}{3}$, we immediately see that $\mathrm{d} \omega_{1}=0$ and that the reduced EDS is still involutive, but now with $s_{1}=2$. Since $t_{0} \neq-1$, the corresponding Lagrangians must locally be of the form $L_{4}(\mathbf{u}, \Sigma)$ as in Example 6.12 by Proposition 6.13. Moreover, these Lagrangians are locally the products $\mathcal{S}^{1} \times P$ for some surface $P$, which is equivalent to saying that $N_{2} \Sigma$ is trivial. Furthermore, by (26), we see that $t_{0}=-\frac{1}{3}$ if and only if the torsion $k_{1}$ of $\Sigma$ satisfies $\left|k_{1}\right|=1$. As observed in 3.2 , this occurs if and only if $\Sigma$ lies linearly full in a totally geodesic $\mathcal{S}^{5}$.

At this point we make an aside concerning austere Lagrangians in $\mathcal{S}^{6}$.

Definition 6.17. Let $L$ be a 3 -dimensional submanifold of $\mathcal{S}^{6}$ and let $h_{L}$ be its second fundamental form. For each $p \in L$, let $\left\{\mathbf{e}_{1}(p), \mathbf{e}_{2}(p), \mathbf{e}_{3}(p)\right\}$ and $\left\{\mathbf{e}_{1}^{\perp}(p), \mathbf{e}_{2}^{\perp}(p)\right.$, $\left.\mathbf{e}_{3}^{\perp}(p)\right\}$ be orthonormal bases for $T_{p} L$ and $N_{p} L$, respectively, and let $2 \omega_{i}$ be the dual 1-form to $\mathbf{e}_{i}$. We can locally write $h_{L}$ using summation notation as

$$
h_{L}=4 h_{i j k} \mathbf{e}_{i}^{\perp} \otimes \omega_{j} \omega_{k}
$$

for some tensor of functions $h_{i j k}$ satisfying $h_{i j k}=h_{i k j}$. Let $q_{i}=4 h_{i j k} \omega_{j} \omega_{k}$ in summation notation. We say that $L$ is austere if, for all $i$, the set of eigenvalues of $q_{i}$ is of the form $\left\{0, \pm \lambda_{i}\right\}$ for some $\lambda_{i}$.

Austere submanifolds were introduced in 14 in the discussion of special Lagrangian submanifolds. Notice that austere 3 -folds in $\mathcal{S}^{6}$ are minimal. A complete classification of austere 3 -folds in $\mathcal{S}^{6}$ is not known, but steps in this direction are taken in [5] and [15. However, we are able to show the following.

Proposition 6.18. A Lagrangian in $\mathcal{S}^{6}$ is austere if and only if its fundamental cubic is either zero or has an $\mathrm{S}_{3}$-stabilizer at each point. Thus, austere Lagrangians in $\mathcal{S}^{6}$ are either simple or given by Proposition 6.13.

Proof. Let $L$ be a Lagrangian in $\mathcal{S}^{6}$ and let $C_{L}$ be its fundamental cubic. Clearly a simple Lagrangian is austere, so assume $L$ is non-simple, so that $C_{L} \neq 0$. Recall the cubic $C(r, s, a, b)$ defined in Lemma 4.2. By a result of Vrancken 24, there exists a frame on $L$ such that $C_{L}=C(r, s, a, b)$ for some functions $r, s, a, b$. Moreover, if $s=0$ we can choose $b=0$. (The key difference between the result in 24] and Lemma 4.2 is that $r$ has to be constant if $L$ is ruled or quasi-ruled.)

We can calculate the quadratic forms $q_{i}$ as in Definition 6.17 as follows:

$$
\begin{aligned}
& q_{1}=2 r \omega_{1}^{2}-(r-s) \omega_{2}^{2}-(r+s) \omega_{3}^{2} ; \\
& q_{2}=-2(r-s) \omega_{1} \omega_{2}+a\left(\omega_{2}^{2}-\omega_{3}^{2}\right)+2 b \omega_{2} \omega_{3} ; \\
& q_{3}=-2(r+s) \omega_{1} \omega_{2}+b\left(\omega_{2}^{2}-\omega_{3}^{2}\right)-2 a \omega_{2} \omega_{3} .
\end{aligned}
$$


Thus, $L$ is austere only if $r\left(r^{2}-s^{2}\right),(r-s)^{2} a$ and $(r+s)^{2} b$ are all zero. Therefore, $C_{L}$ is, up to a choice of frame, $C(r, r, a, 0)$ or $C(0,0, a, 0)$. By Table 1, these cubics have pointwise $\mathrm{S}_{3}$-stabilizers.

6.5. $\mathbb{Z}_{3}$. Suppose that $C_{L} \neq 0$ has a $\mathbb{Z}_{3}$-stabilizer at each point. Therefore, there exist an open dense subset $L^{*}$ of $L$ and functions $r, a: L^{*} \rightarrow \mathbb{R}^{+}$, with $a \neq r \sqrt{2}$, such that $C_{L}=r \omega_{1}\left(2 \omega_{1}^{2}-3 \omega_{2}^{2}-3 \omega_{3}^{2}\right)+a \omega_{2}\left(\omega_{2}^{2}-3 \omega_{3}^{2}\right)$ defines a $\mathbb{Z}_{3}$-subbundle $\mathcal{F}$ of the adapted frame bundle over $L^{*}$.

Calculating the Ricci tensor on $L^{*}$, using Definition 5.2 and Proposition [5.5, we find that it has repeated eigenvalues, so $L$ is quasi-Einstein. Furthermore, Table 2 shows that $L$ is non-simple and quasi-Einstein if and only if $C_{L}$ has a pointwise $\mathrm{SO}(2)-, \mathrm{A}_{4^{-}}, \mathrm{S}_{3^{-}}$or $\mathbb{Z}_{3}$-stabilizer. As mentioned before, quasi-Einstein Lagrangians in $\mathcal{S}^{6}$ are classified in [6], so we can give the remaining examples.

Example 6.19 (Quasi-ruled tubes in the second normal bundle). Let $\mathbf{u}: \Sigma \rightarrow \mathcal{S}^{6}$ be a null-torsion pseudoholomorphic curve with no totally geodesic points. Let $h_{\Sigma}$ be the second fundamental form of $\Sigma$ and let $\mathcal{U}(\Sigma)$ be its unit tangent bundle. Define $\mathbf{x}: \mathcal{U}(\Sigma) \rightarrow \mathcal{S}^{6}$ by

$$
\mathbf{x}: \mathbf{t} \mapsto \frac{\sqrt{5}}{3} \mathbf{u}+\frac{2}{3} \mathbf{t} \times \frac{h_{\Sigma}(\mathbf{t}, \mathbf{t})}{\left\|h_{\Sigma}(\mathbf{t}, \mathbf{t})\right\|}
$$

By [6, Theorem 1], $\mathbf{x}$ is a Lagrangian immersion. If $\Sigma$ has isolated totally geodesic points, $\mathbf{x}$ defines an immersion on an open dense subset of $\mathcal{U}(\Sigma)$ and its image $L_{5}(\mathbf{u}, \Sigma)$ is Lagrangian in $\mathcal{S}^{6}$. Moreover, $L_{5}(\mathbf{u}, \Sigma)$ is quasi-Einstein and does not satisfy Chen's equality. We deduce from Table 2, Lemma 6.9, and Propositions 6.5. 6.7 and 6.13 that $L_{5}(\mathbf{u}, \Sigma)$ has a fundamental cubic with a $\mathbb{Z}_{3}$-stabilizer as long as $\Sigma \neq \mathcal{S}^{2}\left(\frac{1}{6}\right)$.

From Definition 4.7 and (9), we quickly see that $L_{5}(\mathbf{u}, \Sigma)$ is a tube of radius $\sin ^{-1}\left(\frac{2}{3}\right)$ in $N_{2} \Sigma$ about $\Sigma$, and thus is quasi-ruled.

Remark. The Lagrangian $L_{2}$ given in Example 6.6, whose fundamental cubic has an $\mathrm{A}_{4}$-stabilizer, is $L_{5}(\mathbf{u}, \Sigma)$, where $\Sigma$ is the Borůva sphere in $\mathcal{S}^{6}$.

Combining [6, Theorems $1 \& 2$ ] and our observations thus far, we get the next result. Notice that the constant curvature null-torsion pseudoholomorphic curves are totally geodesic 2 -spheres and $\mathcal{S}^{2}\left(\frac{1}{6}\right)$.

Proposition 6.20. A connected, non-simple, Lagrangian $L$ in $\mathcal{S}^{6}$ has a fundamental cubic with a $\mathbb{Z}_{3}$-stabilizer at each point if and only if there exists an open dense subset $L^{*}$ of $L$ such that, for every point $x \in L^{*}$, there exist an open set $U \ni x$ and a null-torsion, non-constant curvature, pseudoholomorphic curve $\mathbf{u}: \Sigma \rightarrow \mathcal{S}^{6}$ with $U \cap L^{*}=L_{5}(\mathbf{u}, \Sigma)$ as given in Example 6.19.

Now, for possible interest, we record the structure equations. Recall that we have a $\mathbb{Z}_{3}$-subbundle $\mathcal{F}$ of the adapted frame bundle over an open dense subset $L^{*}$ of $L$. We deduce from (6) that $r=2 \sqrt{5}$, so we can take $L^{*}=L$ and $C_{L}=$ $2 \sqrt{5} \omega_{1}\left(2 \omega_{1}^{2}-3 \omega_{2}^{2}-3 \omega_{3}^{2}\right)+a \omega_{2}\left(\omega_{2}^{2}-3 \omega_{3}^{2}\right)$. Further, there exist functions $t_{2}, t_{3}$ on $\mathcal{F}$ such that

$$
\alpha_{1}=-\frac{1}{2} \omega_{1}+t_{3} \omega_{2}-t_{2} \omega_{3} ; \quad \alpha_{2}=-\frac{1}{2} \omega_{2} ; \quad \alpha_{3}=-\frac{1}{2} \omega_{3},
$$


and

$$
\mathrm{d} a=-3 a\left(t_{2} \omega_{2}+t_{3} \omega_{3}\right) .
$$

We may therefore write the structure equations as

$$
\begin{aligned}
& \mathrm{d} \omega_{1}=\omega_{2} \wedge \omega_{3} ; \\
& \mathrm{d} \omega_{2}=-t_{3} \omega_{2} \wedge \omega_{3}+\omega_{3} \wedge \omega_{1} ; \\
& \mathrm{d} \omega_{3}=t_{2} \omega_{2} \wedge \omega_{3}+\omega_{1} \wedge \omega_{2} .
\end{aligned}
$$

Furthermore, there exist functions $u_{2}$ and $u_{3}$ such that

$$
\begin{aligned}
& \mathrm{d} t_{2}=-t_{3} \omega_{1}+\left(\frac{1}{16} a^{2}+u_{2}-\frac{1}{2}\left(t_{2}^{2}+t_{3}^{2}+5\right)\right) \omega_{2}+u_{3} \omega_{3} \\
& \mathrm{~d} t_{3}=t_{2} \omega_{1}+u_{3} \omega_{2}+\left(\frac{1}{16} a^{2}-u_{2}-\frac{1}{2}\left(t_{2}^{2}+t_{3}^{2}+5\right)\right) \omega_{3} .
\end{aligned}
$$

Remark. We can interpret a result of Fox [12, Theorem 9.3] as follows: a coassociative cone $N_{0}$ on a Lagrangian in $\mathcal{S}^{6}$ whose fundamental cubic has a $\mathbb{Z}_{3}$-stabilizer is the limit, as $t \rightarrow 0$, of a family of non-singular coassociative 4 -folds $N_{t}$ in $\operatorname{Im} \mathbb{O}$, which are asymptotically conical to $N_{0}$ at infinity.

6.6. $\mathbb{Z}_{\mathbf{2}}$. In second order family studies similar to the type considered here, the $\mathbb{Z}_{2}$ case is typically the most complicated and hardest to classify. However, for Lagrangians in $\mathcal{S}^{6}$, it could not be easier [26, Theorem 2].

Proposition 6.21. The only connected Lagrangian submanifolds of $\mathcal{S}^{6}$ whose fundamental cubic has a $\mathbb{Z}_{2}$-stabilizer at each point are simple.

6.7. Summary. We have shown that a Lagrangian $L$ in $\mathcal{S}^{6}$ whose fundamental cubic has a non-trivial stabilizer in $\mathrm{SO}(3)$ at each point is either ruled or quasiruled. Moreover, $L$ is a Hopf lift to $\mathcal{S}^{5} \subseteq \mathcal{S}^{6}$ of a holomorphic curve in $\mathbb{C P}^{2}$, or given locally as a tube about a pseudoholomorphic curve $\Sigma$. Further, the tube is in $N_{2} \Sigma$ if $\Sigma$ is non-totally geodesic. Thus, we can summarise our results by associating to each non-trivial stabilizer a holomorphic curve or a pseudoholomorphic curve and a tube radius. This is the content of Table 3 below.

TABle 3. Summary of examples as Hopf lifts of holomorphic curves in $\mathbb{C P}^{2}$ and tubes about pseudoholomorphic curves in $\mathcal{S}^{6}$

\begin{tabular}{|c|c|c|c|}
\hline Stabilizer & Holomorphic curve & Pseudoholomorphic curve & Tube radius \\
\hline $\mathrm{SO}(3)$ & Totally geodesic & Totally geodesic & $\frac{\pi}{2}$ \\
$\mathrm{SO}(2)$ & & Totally geodesic & $\sin ^{-1}\left(\frac{2}{3}\right)$ \\
$\mathrm{A}_{4}$ & & Null-torsion $\mathcal{S}^{2}\left(\frac{1}{6}\right)$ & $\sin ^{-1}\left(\frac{2}{3}\right)$ \\
$\mathrm{S}_{3}$ & Non-totally geodesic & Non-totally geodesic & $\frac{\pi}{2}$ \\
$\mathbb{Z}_{3}$ & & Null-torsion not $\mathcal{S}^{2}\left(\frac{1}{6}\right)$ & $\sin ^{-1}\left(\frac{2}{3}\right)$ \\
$\mathbb{Z}_{2}$ & Totally geodesic & Totally geodesic & $\frac{\pi}{2}$ \\
\hline
\end{tabular}


Remark. For the stabilizer $\mathrm{G} \neq \mathrm{S}_{3}$ or $\mathbb{Z}_{3}$, the examples in Table 3 are rigid; i.e. they are unique up to $\mathrm{G}_{2}$ transformations of $\mathcal{S}^{6}$. However, the $\mathrm{S}_{3}$ and $\mathbb{Z}_{3}$ examples have non-trivial deformations given by deformations of the underlying curve.

\section{The RUled LAGRANGIAN FAMILY}

Before we discuss the general ruled family, we prove the following result.

Proposition 7.1. Any connected Lagrangian in $\mathcal{S}^{6}$ with two distinct 1-rulings is simple.

Proof. If a connected Lagrangian $L$ in $\mathcal{S}^{6}$ has two distinct 1-rulings, then the cone $N$ on $L$ in $\operatorname{Im} \mathbb{O}$ is coassociative with two distinct 2-rulings by Lemma 4.4 In $\mathbb{O} \cong \mathbb{R}^{8}$ there are calibrated 4-dimensional submanifolds called Cayley 4-folds. If we embed $N$ in $\mathbb{O}=\mathbb{R} \oplus \operatorname{Im} \mathbb{O}$ as $C=\{0\} \times N$, then $C$ is a 2-ruled Cayley cone by [20, Proposition 2.11]. Moreover, $C$ has two distinct 2-rulings and so, by [20, Proposition 4.4], must be a 4-plane. Thus, $N$ is a 4 -plane and $L$ is simple, as claimed.

Remark. This is the analogue of [3, Theorem 6 part 4], and we could have proved it in an analogous manner. The key points are that a Lagrangian $L$ has two distinct 1-rulings if and only if $C_{L}$ has an $\mathrm{A}_{4^{-}}$or $\mathbb{Z}_{2^{-}}$-stabilizer and that the only ruled Lagrangians such that $C_{L}$ has an $\mathrm{A}_{4^{-}}$or $\mathbb{Z}_{2}$-stabilizer are simple by Table 3 ,

Let $L$ be a Lagrangian in $\mathcal{S}^{6}$ ruled by geodesic circles. By Lemma 4.2, using the notation there, we can choose a frame on $L$ such that the fundamental cubic $C_{L}=C(0, s, a, b)$ for some functions $s, a, b$. We are interested in the possibility of ruled Lagrangian submanifolds $L$ for which $C_{L}$ does not have a pointwise symmetry, so we make this assumption.

As stated in Lemma 4.2. if $s=0$ we can choose $b=0$. However, $C(0,0, a, 0)$ has at least an $\mathrm{S}_{3}$-stabilizer at each point by Table 1 . We also notice from Table 1 that $C(0, s, 0,0)$ has a pointwise symmetry. Thus, we assume that $s$ and $a^{2}+b^{2}$ are both non-zero on some open dense subset $L^{*}$ of $L$.

Using (6) we calculate

$$
\begin{aligned}
\alpha_{1}= & t_{0} \omega_{1}+t_{3} \omega_{2}-t_{2} \omega_{3} \\
\alpha_{2}= & -\left(1+t_{0}\right) \omega_{2}+t_{1} \omega_{3} \\
\alpha_{3}= & -t_{1} \omega_{2}-\left(1+t_{0}\right) \omega_{3} ; \\
\mathrm{d} s= & -2 s\left(t_{1} \omega_{1}+t_{2} \omega_{2}+t_{3} \omega_{3}\right) \\
\mathrm{d} a= & -\left(2 s t_{2}+a t_{1}+b\left(1+2 t_{0}\right)\right) \omega_{1}+\left(c_{1}-s t_{1}-\frac{3}{2} a t_{2}-\frac{3}{2} b t_{3}\right) \omega_{2} \\
& +\left(c_{2}-\left(1+2 t_{0}\right) s-\frac{3}{2} a t_{3}-\frac{3}{2} b t_{2}\right) \omega_{3} ; \\
\mathrm{d} b= & -\left(2 s t_{3}-a\left(1+2 t_{0}\right)+b t_{1}\right) \omega_{1}+\left(c_{2}+\left(1+2 t_{0}\right) s+\frac{3}{2} a t_{3}+\frac{3}{2} b t_{2}\right) \omega_{2} \\
& -\left(c_{1}+s t_{1}+\frac{3}{2} a t_{2}+\frac{3}{2} b t_{3}\right) \omega_{3}
\end{aligned}
$$


for some functions $t_{0}, t_{1}, t_{2}, t_{3}, c_{1}, c_{2}$. Thus, the structure equations are

$$
\begin{aligned}
\mathrm{d} \omega_{1} & =-2 t_{0} \omega_{2} \wedge \omega_{3} ; \\
\mathrm{d} \omega_{2} & =-t_{3} \omega_{2} \wedge \omega_{3}+\omega_{3} \wedge \omega_{1}+t_{1} \omega_{1} \wedge \omega_{2} ; \\
\mathrm{d} \omega_{3} & =t_{2} \omega_{2} \wedge \omega_{3}-t_{1} \omega_{3} \wedge \omega_{1}+\omega_{1} \wedge \omega_{2} .
\end{aligned}
$$

Moreover, there exist functions $u_{1}, u_{2}, u_{3}, u_{4}$ such that

$$
\begin{aligned}
\mathrm{d} t_{0} & =-2 t_{0} t_{1} \omega_{1}+\left(u_{1}-\frac{1}{16} s b\right) \omega_{2}+\left(\frac{1}{16} s a+u_{2}\right) \omega_{3} \\
\mathrm{~d} t_{1} & =\left(\frac{1}{16} s^{2}+t_{0}^{2}-t_{1}^{2}-4\right) \omega_{1}+\left(\frac{1}{16} s a-u_{2}\right) \omega_{2}+\left(u_{1}+\frac{1}{16} s b\right) \omega_{3} ; \\
\mathrm{d} t_{2} & =\left(\frac{1}{16} s a-t_{3}-t_{1} t_{2}-u_{2}\right) \omega_{1}+\left(u_{4}-t_{0} t_{1}\right) \omega_{3} \\
& +\left(\frac{1}{2}\left(\frac{1}{16} s^{2}+\frac{1}{8} a^{2}+\frac{1}{8} b^{2}-t_{0}\left(2+3 t_{0}\right)-t_{1}^{2}-t_{2}^{2}-t_{3}^{2}-4\right)+u_{3}\right) \omega_{2} ; \\
\mathrm{d} t_{3} & =\left(\frac{1}{16} s b+t_{2}-t_{3} t_{1}+u_{1}\right) \omega_{1}+\left(u_{4}+t_{0} t_{1}\right) \omega_{2} \\
& +\left(\frac{1}{2}\left(\frac{1}{16} s^{2}+\frac{1}{8} a^{2}+\frac{1}{8} b^{2}-t_{0}\left(2+3 t_{0}\right)-t_{1}^{2}-t_{2}^{2}-t_{3}^{2}-4\right)-u_{3}\right) \omega_{3} .
\end{aligned}
$$

Setting up the appropriate EDS here, we find that it is involutive with its last non-zero Cartan character $s_{1}=6$. Thus, ruled Lagrangian submanifolds of $\mathcal{S}^{6}$ depend locally on 6 functions of 1 variable. The largest ruled family we have seen so far (Example 6.12) depends only on 4 functions of 1 variable locally, so there must be another family describing the general ruled Lagrangians.

We shall describe a family of ruled Lagrangians in $\mathcal{S}^{6}$ with the "right" local dependence on functions of one variable, and then prove that this family provides a local classification for the generic ruled Lagrangian. We start with a definition.

Definition 7.2. Let $\mathbf{u}: \Sigma \rightarrow \mathcal{S}^{6}$ be a pseudoholomorphic curve and use the notation of 3.2 In particular, denote a special unitary frame for $\mathbf{u}^{*}\left(T \mathcal{S}^{6}\right)$ by $\left\{\mathbf{f}_{1}, \mathbf{f}_{2}, \mathbf{f}_{3}\right\}$ such that $\mathbf{f}_{1}$ spans $T^{1,0} \Sigma$ and $\left\{\mathbf{f}_{2}, \mathbf{f}_{3}\right\}$ is a basis for $N \Sigma$. Let $\mathcal{B}(\Sigma)$ be the $\mathrm{U}(2)$-bundle of such frames $\left\{\mathbf{f}_{1}, \mathbf{f}_{2}, \mathbf{f}_{3}\right\}$ over $\Sigma$.

We define two $\mathrm{U}(1)$ actions on $\mathcal{B}(\Sigma)$. The first, $\mathrm{U}(1)_{l}$, is the action which fixes $\mathbf{f}_{3}$ :

$$
\left(\mathbf{f}_{1}, \mathbf{f}_{2}, \mathbf{f}_{3}\right) \longmapsto\left(e^{i t} \mathbf{f}_{1}, e^{-i t} \mathbf{f}_{2}, \mathbf{f}_{3}\right) .
$$

The second, $\mathrm{U}(1)_{r}$, is the rotation of $\mathbf{f}_{3}$ :

$$
\left(\mathbf{f}_{1}, \mathbf{f}_{2}, \mathbf{f}_{3}\right) \longmapsto\left(e^{-i t} \mathbf{f}_{1}, e^{-i t} \mathbf{f}_{2}, e^{2 i t} \mathbf{f}_{3}\right) .
$$

Let $\mathcal{Q}(\Sigma)=\mathcal{B}(\Sigma) / \mathrm{U}(1)_{l}$ and let $\mathcal{X}(\Sigma)=\mathcal{Q}(\Sigma) / \mathrm{U}(1)_{r}$. Note that we have a projection $\pi_{\mathcal{X}}: \mathcal{Q}(\Sigma) \rightarrow \mathcal{X}(\Sigma)$ whose fibres are circles.

Clearly, surfaces in the 4-manifold $\mathcal{X}(\Sigma)$ lift to 3-dimensional submanifolds of $\mathcal{Q}(\Sigma)$ via $\pi_{\mathcal{X}}$. Moreover, these 3-folds can be thought of as tubes in $N \Sigma$ about $\Sigma$. Hence, the tubes of this type which are Lagrangian are equivalent to distinguished surfaces in $\mathcal{X}(\Sigma)$. This motivates the following key result.

Theorem 7.3. Let $\mathbf{u}: \Sigma \rightarrow \mathcal{S}^{6}$ be a non-totally geodesic pseudoholomorphic curve and use the notation of Definition 7.2. There is an integrable complex structure I on $\mathcal{X}(\Sigma)$ such that $\mathcal{X}(\Sigma)$ is a holomorphic $\mathbb{C P}^{1}$-bundle over $\Sigma$, and a (real) surface $\Gamma$ in $\mathcal{X}(\Sigma)$ is a holomorphic curve if and only if the image of the map $\mathbf{x}: \pi_{\mathcal{X}}^{-1}(\Gamma) \rightarrow \mathcal{S}^{6}$ given by $\mathbf{x}=\mathbf{f}_{3}+\overline{\mathbf{f}}_{3}$ is a ruled Lagrangian in $\mathcal{S}^{6}$. 
Proof. Define $\mathbf{x}: \mathcal{Q}(\Sigma) \rightarrow \mathcal{S}^{6}$ by $\mathbf{x}=\mathbf{f}_{3}+\overline{\mathbf{f}}_{3}$. This map is certainly well-defined on $\mathcal{Q}(\Sigma)$, since it is defined on $\mathcal{B}(\Sigma)$ and the action of $\mathrm{U}(1)_{l}$ fixes $\mathbf{f}_{3}$. Using the structure equations (11) for the adapted frame bundle over $\Sigma$, we see that

$$
\mathrm{d} \mathbf{x}=-\bar{k}_{3} \mathbf{f}_{1} \bar{\theta}_{1}-k_{3} \overline{\mathbf{f}}_{1} \theta_{1}-\mathbf{f}_{2}\left(\bar{\kappa}_{32}-\bar{\theta}_{1}\right)-\overline{\mathbf{f}}_{2}\left(\kappa_{32}-\theta_{1}\right)+\left(\mathbf{f}_{3}-\overline{\mathbf{f}}_{3}\right) \kappa_{33},
$$

where we remind the reader that $\theta_{1}$ is the dual 1 -form to $\mathbf{f}_{1}, \kappa$ is a $3 \times 3$ traceless skew-Hermitian matrix and $k_{3}$ is a holomorphic function. Therefore, from (8),

$$
\mathbf{x} \times \mathrm{d} \mathbf{x}=-i \mathbf{u} \kappa_{33}+\mathbf{f}_{1}\left(\kappa_{32}-\theta_{1}\right)+\overline{\mathbf{f}}_{1}\left(\bar{\kappa}_{32}-\bar{\theta}_{1}\right)-k_{3} \mathbf{f}_{2} \theta_{1}-\bar{k}_{3} \overline{\mathbf{f}}_{2} \bar{\theta}_{1} .
$$

Recall Definition 2.3. In particular, the almost complex structure $J$ on $\mathcal{S}^{6}$ is given by a cross product with the position vector on $\mathcal{S}^{6}$ and the almost symplectic form $\omega$ on $\mathcal{S}^{6}$ is given by $\omega(\mathbf{u}, \mathbf{v})=J \mathbf{u} \cdot \mathbf{v}$. Thus the pull-back of $\omega$ by $\mathbf{x}$ is given by $\mathbf{x}^{*}(\omega)=(\mathbf{x} \times \mathrm{d} \mathbf{x}) \cdot \mathrm{d} \mathbf{x}$, and we deduce that

$$
\check{\omega}=\frac{1}{4} \mathbf{x}^{*}(\omega)=\operatorname{Re}\left(\kappa_{32} \wedge k_{3} \theta_{1}\right) .
$$

Again using (11), we calculate that

$$
\mathrm{d} \check{\omega}=-3 i \kappa_{33} \wedge \check{\Upsilon}, \quad \text { where } \check{\Upsilon}=\operatorname{Im}\left(\kappa_{32} \wedge k_{3} \theta_{1}\right) .
$$

Therefore, we are led to define the 2 -form $\check{\Omega}$ on $\mathcal{Q}(\Sigma)$ by

$$
\check{\Omega}=\check{\omega}+i \check{\Upsilon}=\kappa_{32} \wedge k_{3} \theta_{1} \text {. }
$$

By (11), it is clear that

$$
\mathrm{d} \check{\Omega}=-3 \kappa_{33} \wedge \check{\Omega} .
$$

Equation (36) shows that $\check{\Omega}$ pushes down to $\mathcal{X}(\Sigma)$. We also see, from (33)-(35), that $\mathbf{x}: L^{3} \subseteq \mathcal{Q}(\Sigma) \rightarrow \mathcal{S}^{6}$ is a Lagrangian immersion if and only if $\left.\check{\Omega}\right|_{L} \equiv 0$.

Since $\Sigma$ is non-totally geodesic we can assume that $k_{3}$ only has isolated zeros. Further, as we have not adapted frames so that $\mathbf{f}_{2}$ and $\mathbf{f}_{3}$ span $N_{1} \Sigma$ and $N_{2} \Sigma, \kappa_{32}$ is a non-zero 1-form independent of $\theta_{1}$. Thus, $\check{\Omega}$ is a definite 2 -form on an open dense subset of $\mathcal{X}(\Sigma)$. Hence, by (36), $\check{\Omega}$ defines an integrable complex structure $I$ on $\mathcal{X}(\Sigma)$. Notice, trivially, that the $(1,0)$-form $\theta_{1}$ on $\Sigma$ pulls back to $\mathcal{X}(\Sigma)$ to be a linear combination of $\kappa_{32}$ and $k_{3} \theta_{1}$, away from the zeroes of $k_{3}$. Thus, the projection from $\mathcal{X}(\Sigma)$ to $\Sigma$ is holomorphic and $\mathcal{X}(\Sigma)$ is a $\mathbb{C P}^{1}$-bundle over $\Sigma$. Moreover, $I$ has the property that a real surface $\Gamma$ in $\mathcal{X}(\Sigma)$ is a holomorphic curve if and only if $L=\pi_{\mathcal{X}}^{-1}(\Gamma) \subseteq \mathcal{Q}(\Sigma)$ satisfies $\left.\check{\Omega}\right|_{L} \equiv 0$. Since $L$ is clearly ruled, the result follows.

If $\Sigma$ is totally geodesic, the proof of Theorem 7.3 shows that every lift of $\Sigma$ to $\mathcal{X}(\Sigma)$ defines a ruled Lagrangian. However, these Lagrangians must be invariant under the $\mathrm{SO}(4)$ subgroup of $\mathrm{G}_{2}$ preserving $\Sigma$ and so are simple.

Remarks. Since $\mathcal{X}(\Sigma)$ is a $\mathbb{C P}^{1}$-bundle over a Riemann surface $\Sigma$, it is a ruled complex surface and so is algebraic by Kodaira's classification of complex surfaces. Therefore, holomorphic curves in $\mathcal{X}(\Sigma)$ are well understood using techniques in algebraic geometry. Moreover, if $\Sigma$ is non-compact, $\mathcal{X}(\Sigma)$ is trivial, and so holomorphic curves in $\mathcal{X}(\Sigma)$ are equivalent to holomorphic functions from $\Sigma$ to $\mathbb{C P}^{1}$.

We now present the most general family of ruled Lagrangians in $\mathcal{S}^{6}$. 
Example 7.4 (The general ruled family). Let $\mathbf{u}: \Sigma \rightarrow \mathcal{S}^{6}$ be a non-totally geodesic pseudoholomorphic curve and use the notation of Definition 7.2, By Theorem 7.3 , the bundle $\mathcal{X}(\Sigma)$ is endowed with an integrable complex structure. Let $\Gamma$ be a holomorphic curve in $\mathcal{X}(\Sigma)$ and define $\mathbf{x}: \pi_{\mathcal{X}}^{-1}(\Gamma) \rightarrow \mathcal{S}^{6}$ by $\mathbf{x}=\mathbf{f}_{3}+\overline{\mathbf{f}}_{3}$. By Theorem 7.3, the image $L_{6}(\mathbf{u}, \Sigma, \Gamma)$ of $\mathbf{x}$ is a ruled Lagrangian.

Let $\Pi$ be the 2-plane bundle over $\Sigma$ defined by $2 i \overline{\mathbf{f}}_{3} \wedge \mathbf{f}_{3}$. By Definition 4.7, $L_{6}(\mathbf{u}, \Sigma, \Gamma)$ is a tube of radius $\frac{\pi}{2}$ in $\Pi$ about $\Sigma$. Furthermore, these examples depend locally on 6 functions of 1 variable since our data consists of a pseudoholomorphic curve in $\mathcal{S}^{6}$ and a holomorphic curve in a complex 2-manifold.

Since $\mathcal{X}(\Sigma)$ is a subbundle of the frame bundle over $\Sigma$, the structure equations on it are given by the $G_{2}$ structure equations (10) for some vector of 1-forms $\theta=\left(\theta_{1}, \theta_{2}, \theta_{3}\right)^{\mathrm{T}}$ and some traceless $3 \times 3$ skew-Hermitian matrix $\kappa$. Moreover, from Definition 7.2, we see that we can adapt frames on $\Sigma$ so that $\theta_{3}=0$ on $\mathcal{X}(\Sigma)$. From the proof of Theorem [7.3] a surface $\Gamma$ in $\mathcal{X}(\Sigma)$ is a holomorphic curve if and only if $\kappa_{31} \wedge \kappa_{32}$ vanishes on $\Gamma$ (recalling that $\kappa_{31}=k_{3} \theta_{1}$ on $\Sigma$ ). Thus, the structure equations for a holomorphic curve $\Gamma$ in $\mathcal{X}(\Sigma)$ are given by (10) with: $\mathbf{u}$ the immersion of $\Sigma$ in $\mathcal{S}^{6} ; \mathbf{f}$ a unitary frame for $\left.T \mathcal{S}^{6}\right|_{\Sigma} ; \theta=\left(\theta_{1}, \theta_{2}, 0\right)$; and $\kappa$ satisfying $\kappa_{31} \wedge \kappa_{32}=0$.

Remark. One can use the formulae (9) for a unitary frame for $\left.T \mathcal{S}^{6}\right|_{\Sigma}$ to give a "more explicit" expression for $L_{6}(\mathbf{u}, \Sigma, \Gamma)$. However, in doing so, one adapts frames so that $\mathbf{f}_{2}$ and $\mathbf{f}_{3}$ span $N_{1} \Sigma$ and $N_{2} \Sigma$. This breaks the symmetry of the problem and makes it difficult to see the holomorphic condition on $\Gamma$ in the formula one derives for the Lagrangian. We therefore refrain from giving this expression for $L_{6}(\mathbf{u}, \Sigma, \Gamma)$.

We now prove the main result in this paper.

Theorem 7.5. A connected Lagrangian $L$ in $\mathcal{S}^{6}$ is ruled if and only if there exists an open dense subset $L^{*}$ of $L$ such that, for all $x \in L^{*}$, there exists an open set $U \ni x$ such that:

(a) $U \cap L^{*}=L_{3}\left(\mathbf{u}_{1}, \Sigma_{1}\right)$ for some holomorphic curve $\mathbf{u}_{1}: \Sigma_{1} \rightarrow \mathbb{C P}^{2}$ as in Example 6.11, and we may take $U=L^{*}=L$; or

(b) $U \cap L^{*}=L_{4}\left(\mathbf{u}_{2}, \Sigma_{2}\right)$ for some non-totally geodesic pseudoholomorphic curve $\mathbf{u}_{2}: \Sigma_{2} \rightarrow \mathcal{S}^{6}$ as in Example 6.12; or

(c) $U \cap L^{*}=L_{6}\left(\mathbf{u}_{3}, \Sigma_{3}, \Gamma\right)$ for some non-totally geodesic pseudoholomorphic curve $\mathbf{u}_{3}: \Sigma_{3} \rightarrow \mathcal{S}^{6}$ and some holomorphic curve $\Gamma$ in $\mathcal{X}\left(\Sigma_{3}\right)$ as in Example 7.4.

Moreover, $L$ is not linearly full if and only if $L$ is of type (a) and the general family of ruled Lagrangians in $\mathcal{S}^{6}$ is locally classified by Example 7.4 .

Note. Theorem 7.5 gives 'Weierstrass formulae' for ruled Lagrangians in $\mathcal{S}^{6}$ in the following sense: we can define them either using a holomorphic curve in $\mathbb{C P}^{2}$ or, given a pseudoholomorphic curve in $\mathcal{S}^{6}$, using holomorphic data. However, we should stress that the general pseudoholomorphic curve in $\mathcal{S}^{6}$ does not admit a Weierstrass representation (though the null-torsion curves do by [2, Theorem 4.7]). Therefore, we really have a family of Weierstrass representations for the general ruled Lagrangian, indexed by pseudoholomorphic curves in $\mathcal{S}^{6}$. This is in stark contrast to ruled special Lagrangians in $\mathbb{C}^{3}$ which, it is believed, cannot be similarly described using Weierstrass formulae. 
Proof. If $L$ has a fundamental cubic $C_{L}$ with pointwise symmetry, then by Proposition 6.13 and the observations at the start of this section it must locally be given by (a) or (b) depending on whether or not it is linearly full. It also follows from Proposition 6.13 that $L$ is of type (a) if and only if $L$ is not linearly full and the local description can be extended to a global one.

Therefore, suppose $C_{L}$ does not have a pointwise symmetry. Then, on some open dense subset $L^{*}$, the structure equations are given by (30)-(32). Recall the notation in $\$ 3.2$ and define

$$
\begin{gathered}
\mathbf{u}=-J \mathbf{e}_{1} ; \\
\mathbf{f}_{1}=\frac{1}{2}\left(-J \mathbf{e}_{2}+i J \mathbf{e}_{3}\right) ; \quad \mathbf{f}_{2}=\frac{1}{2}\left(\mathbf{e}_{2}+i \mathbf{e}_{3}\right) ; \quad \mathbf{f}_{3}=\frac{1}{2}\left(\mathbf{x}+i \mathbf{e}_{1}\right) ; \\
\theta_{1}=\frac{1}{2}\left(\left(t_{0}+2\right)+i t_{1}\right)\left(\omega_{2}+i \omega_{3}\right) ; \quad \theta_{2}=\frac{1}{2}\left(\frac{i s}{4}\right)\left(\omega_{2}+i \omega_{3}\right) ; \\
\kappa_{22}=i\left(\left(1+t_{0}\right) \omega_{1}+t_{3} \omega_{2}-t_{2} \omega_{3}\right) ; \quad \kappa_{33}=-2 i \omega_{1} ; \\
\kappa_{31}=-\theta_{2} ; \quad \kappa_{21}=\frac{s}{4} \omega_{1}+\frac{a-i b}{4}\left(\omega_{2}+i \omega_{3}\right) ; \\
\kappa_{32}=\frac{1}{2}\left(\left(t_{0}-2\right)+i t_{1}\right)\left(\omega_{2}+i \omega_{3}\right) .
\end{gathered}
$$

Let $\theta=\left(\theta_{1}, \theta_{2}, 0\right)^{\mathrm{T}}$ and let $\kappa$ be the $3 \times 3$ traceless skew-Hermitian matrix of 1 -forms defined using (37d)-(37f). Using (30)-(32), we find that $\mathbf{u}, \mathbf{f}=\left(\mathbf{f}_{1} \mathbf{f}_{2} \mathbf{f}_{3}\right), \theta$ and $\kappa$ satisfy the $G_{2}$ structure equations (10).

We observe that $\mathrm{d} \mathbf{u}$ is independent of $\omega_{1}$ and that left-multiplication by $\mathbf{u}$ acts as a complex structure map on $\mathbf{f}=\left(\mathbf{f}_{1} \mathbf{f}_{2} \mathbf{f}_{3}\right)$ (this is easily verified by taking explicit imaginary octonionic representatives). Thus, $\mathbf{u}$ defines a pseudoholomorphic curve $\mathbf{u}: \Sigma \rightarrow \mathcal{S}^{6}$ and $\mathbf{f}$ is a unitary frame for $\mathbf{u}^{*}\left(T \mathcal{S}^{6}\right)$. Moreover, $\kappa_{21} \neq 0$ since $s$ and $a^{2}+b^{2}$ are non-zero functions, so $\Sigma$ must be non-totally geodesic by the observations in $\$ 3.2$. Further, we see that $\kappa_{31} \wedge \kappa_{32}=0$.

By the observations after Example 7.4 the structure equations satisfied by $\mathbf{u}, \mathbf{f}$, $\theta$ and $\kappa$ define a holomorphic curve $\Gamma$ in $\mathcal{X}(\Sigma)$ with respect to the complex structure $I$ given by Theorem 7.3. Since $\mathbf{x}=\mathbf{f}_{3}+\overline{\mathbf{f}}_{3}$, we deduce from Theorem 7.3 that $L^{*}$ is locally of the form $L_{6}(\mathbf{u}, \Sigma, \Gamma)$ as in Example 7.4.

Remark. The general ruled Lagrangian admits two types of deformations: deformations of the underlying pseudoholomorphic curve $\Sigma$ and deformations of the holomorphic curve in $\mathcal{X}(\Sigma)$.

To conclude this section, we study some reductions of the system for ruled Lagrangians.

Example 7.6 (Ruled tubes in the first normal bundle). Let $\mathbf{u}: \Sigma \rightarrow \mathcal{S}^{6}$ be a pseudoholomorphic curve with no totally geodesic points. Let $h_{\Sigma}$ be the second fundamental form of $\Sigma$ and let $\mathcal{U}(\Sigma)$ be its unit tangent bundle. Define $\mathbf{x}: \mathcal{U}(\Sigma) \rightarrow$ $\mathcal{S}^{6}$ by

$$
\mathbf{x}: \mathbf{t} \mapsto \frac{h_{\Sigma}(\mathbf{t}, \mathbf{t})}{\left\|h_{\Sigma}(\mathbf{t}, \mathbf{t})\right\|}
$$

By [25, Theorem 1], $\mathrm{x}$ is a Lagrangian immersion in $\mathcal{S}^{6}$ if and only if $\Sigma$ is nulltorsion. From Definition 4.7 and (9), we recognise these examples as ruled Lagrangian tubes in $N_{1} \Sigma$ about null-torsion pseudoholomorphic curves $\Sigma$. 
The structure equations (30)-(32) for $t_{1}=0$ define an involutive EDS with $s_{1}=2$. By comparing these structure equations with the calculations leading to the proof of [25, Theorem 2], we deduce that the solutions to this reduced system are given by Example 7.6 .

Remark. By [25, Theorem 2], Lagrangians that admit a Killing vector whose integral curves are geodesic circles are classified by Example 6.11, $L_{4}(\mathbf{u}, \Sigma)$ as in Example 6.12 for a null-torsion pseudoholomorphic curve $\Sigma$, and Example 7.6 .

Example 7.7 (Products 2). The structure equations (30)-(32) with $t_{0}=0$ have $\mathrm{d} \omega_{1}=0$, and the reduced EDS is involutive with $s_{1}=2$. Notice that $t_{1}$ must be non-zero by (32). Thus, by Theorem[7.5, the corresponding Lagrangians are locally the products $\mathcal{S}^{1} \times P$ for a surface $P$ in $\mathcal{S}^{6}$, and the Lagrangians are of the form $L_{6}(\mathbf{u}, \Sigma, \Gamma)$ as in Example 7.4. For $L_{6}(\mathbf{u}, \Sigma, \Gamma)$ to be a product, the plane-bundle over $\Sigma$ defined by $\Gamma$ must be trivial. We see from (37) that $t_{0}=0$ if and only if the torsion $k_{1}$ of $\Sigma$ satisfies $\left|k_{1}\right|=1$. Thus, $\Sigma$ is linearly full in a totally geodesic $\mathcal{S}^{5}$ in $\mathcal{S}^{6}$ by observations in 3.2 .

\section{The QUaSi-Ruled Lagrangian FAmily}

Here we prove that the fundamental cubic of a quasi-ruled Lagrangian has a pointwise symmetry; they have already been classified by examples in $₫ 6$.

Theorem 8.1. A connected Lagrangian $L$ in $\mathcal{S}^{6}$ is quasi-ruled if and only if its fundamental cubic has a pointwise $\mathrm{SO}(2)-$-, $\mathrm{A}_{4}$ - or $\mathbb{Z}_{3}$-symmetry. Therefore, $L$ has $a \frac{2}{3}$-ruling and there exists an open dense subset $L^{*}$ of $L$ such that, for all $x \in L^{*}$, there exists an open set $U \ni x$ such that either $U \cap L^{*}=L_{1}$ as given in Example 6.4 or $U \cap L^{*}=L_{5}(\mathbf{u}, \Sigma)$ for some non-totally geodesic null-torsion pseudoholomorphic curve $\mathbf{u}: \Sigma \rightarrow \mathcal{S}^{6}$ as in Example 6.19.

Proof. Suppose that $L$ is a connected Lagrangian submanifold of $\mathcal{S}^{6}$ with a $\lambda$ ruling, for some $\lambda \in(0,1)$. By Lemma 4.2, using the notation there, we can choose a coframe of $L$ such that its fundamental cubic is $C_{L}=C(r, s, a, b)$ for $r=\frac{4}{\lambda} \sqrt{1-\lambda^{2}} \neq 0$ and some functions $s, a, b$. If $C_{L}$ has a pointwise symmetry we are done by Tables 1 and 3 , so, for a contradiction, we assume otherwise.

If $s=0$, we can choose $b=0$ by Lemma 4.2, but $C(r, 0, a, 0)$ has a pointwise symmetry by Table 1. We also notice that $C(r, s, 0,0)$ has a pointwise symmetry, so $s \neq 0$ and $a^{2}+b^{2} \neq 0$ on some open dense subset $L^{*}$ of $L$.

As $C_{L}$ has a trivial stabilizer in $\mathrm{SO}(3)$ at each point, there exist functions $t_{i j}$ on $L^{*}$ such that $\alpha_{i}=t_{i j} \omega_{j}$. Since $L$ is quasi-ruled in the $\mathbf{e}_{1}$ direction, $t_{21}=t_{31}=0$. From the structure equations (6a), (6b) and (6d) we find that

$$
\begin{gathered}
s\left(2 t_{11}+1\right)=-(2 r+s)\left(2 t_{22}+1\right)=(2 r-s)\left(2 t_{33}+1\right) \quad \text { and } \\
(2 r+s) t_{23}=(2 r-s) t_{32} .
\end{gathered}
$$

Therefore, we can split our discussion into two cases: $|s|=2 r$ and $|s| \neq 2 r$.

Suppose $|s|=2 r$ and further, without loss of generality, that $s=-2 r$. Equations (38) imply that $t_{11}=t_{33}=-\frac{1}{2}$ and $t_{32}=0$. We also find, since $s$ is constant, that

$$
8 r t_{12}=-a\left(2 t_{22}+1\right)-2 b t_{23} \text { and } 8 r t_{13}=-2 a t_{23}+b\left(2 t_{22}+1\right) .
$$

Putting this information in (6c) leads to $r^{2}=4$, so $r=2$, and

$$
2 t_{12} t_{23}-t_{13}\left(2 t_{22}+1\right)-b=0 \text {. }
$$


From (39)-(40), we deduce that $b=0$ and that $t_{12}$ and $t_{13}$ are multiples, depending on $a$, of $2 t_{22}+1$ and $t_{13}$, respectively. Further, we see that $a$ is necessarily constant and, since

$$
\mathrm{d} a=3\left(1-\frac{1}{16} a^{2}\right)\left(2 t_{23} \omega_{2}-\left(2 t_{22}+1\right) \omega_{3}\right),
$$

we must have that $a^{2}=16$ or $2 t_{22}+1=t_{23}=0$. The former case quickly leads to a contradiction from (6d), whereas the latter forces $a=0$, contradicting our assumption that $a^{2}+b^{2} \neq 0$.

Hence we turn our attention to the possibility of $|s| \neq 2 r$ on $L^{*}$. By (38), there exist functions $t_{0}$ and $t_{1}$ on $L^{*}$ such that

$$
\begin{gathered}
2 t_{11}+1=4\left(4 r^{2}-s^{2}\right) t_{0} ; \quad 2 t_{22}+1=-4 s(2 r-s) t_{0} ; \quad 2 t_{33}+1=4 s(2 r+s) t_{0} \\
t_{23}=2 s(2 r-s) t_{1}, \quad \text { and } \quad t_{32}=2 s(2 r+s) t_{1} .
\end{gathered}
$$

One quickly finds from (6c) that $s$ satisfies $r^{2}+s^{2}=20$, so $s$ is constant. This then forces $t_{1}=0, t_{12}=-4 r a t_{0}$ and $t_{13}=4 r b t_{0}$. Putting this information back in the structure equations (6c)-(6d) leads to $s=b=0$. This is again a contradiction, and the theorem is proved.

We conclude with the analogue of Proposition 7.1 .

Proposition 8.2. A connected quasi-ruled Lagrangian has a unique $\frac{2}{3}$-ruling.

Proof. By Theorem 8.1 a connected quasi-ruled Lagrangian $L$ has a $\frac{2}{3}$-ruling. Moreover, the fundamental cubic $C_{L}$ has $\mathrm{SO}(2)-, \mathrm{A}_{4^{-}}$or $\mathbb{Z}_{3}$-symmetry. Suppose a unit tangent vector $\mathbf{e}$ on $L$ is in the direction of a $\frac{2}{3}$-ruling. Then $h_{L}(\mathbf{e}, \mathbf{e})=2 \sqrt{5} J \mathbf{e}$, where $h_{L}$ is the second fundamental form of $L$. By the work in $\$ 6$, using the notation of Lemma 4.2, $C_{L}=C(2 \sqrt{5}, 0, a, 0)$ for some non-negative function $a$, up to $\mathrm{SO}(3)$ transformation. Elementary algebra then shows that there is a unique vector $\mathbf{e}$ in each case, namely $\mathbf{e}=\mathbf{e}_{1}$ in this frame.

\section{ACKNOWLEDGEMENTS}

The author thanks Robert Bryant and Daniel Fox for useful conversations, and MSRI for its hospitality during the time of this research. The author was supported by an NSF Mathematical Sciences Postdoctoral Research Fellowship.

\section{REFERENCES}

[1] J. Bolton, L. Vrancken and L. M. Woodward, On Almost Complex Curves in the Nearly Kähler 6-Sphere, Q. J. Math. 45 (1994), 407-427. MR.1315456 (96k:53091)

[2] R. L. Bryant, Submanifolds and Special Structures on the Octonions, J. Differential Geom. 17 (1982), 185-232. MR664494 (84h:53091)

[3] R. L. Bryant, Second Order Families of Special Lagrangian 3-folds in $\mathbb{C}^{3}$, in Perspectives in Riemannian Geometry, CRM Proceedings and Lecture Notes, edited by Vestislav Apostolov, Andrew Dancer, Nigel Hitchin, and McKenzie Wang, 40 (2006), American Mathematical Society. MR2237106 (2007e:53063)

[4] B. Y. Chen, Some Pinching and Classification Theorems for Minimal Submanifolds, Arch. Math. (Basel) 60 (1993), 568-578. MR1216703 (94d:53093)

[5] M. Dajczer and L. Florit, A Class of Austere Submanifolds, Illinois J. Math. 45 (2001), 735-755. MR.1879232 (2003g:53090)

[6] R. Deszcz, F. Dillen, L. Verstraelen and L. Vrancken, Quasi-Einstein Totally Real Submanifolds of the Nearly Kähler 6-Sphere, Tohoku Math. J. 51 (1999), 461-478. MR.1725622 (2001a:53084) 
[7] F. Dillen, L. Verstraelen and L. Vrancken, Classification of Totally Real 3-Dimensional Submanifolds of $\mathcal{S}^{6}(1)$ with $K \geq 1 / 16$, J. Math. Soc. Japan 42 (1990), 565-584. MR 1069845 (91k:53064)

[8] F. Dillen and L. Vrancken, Totally Real Submanifolds in $\mathcal{S}^{6}(1)$ Satisfying Chen's Equality, Trans. Amer. Math. Soc. 348 (1996), 1633-1646. MR.1355070 (96h:53070)

[9] N. Ejiri, Totally Real Submanifolds in a 6-Sphere, Proc. Amer. Math. Soc. 83 (1981), 759763. MR630028 (83a:53033)

[10] N. Ejiri, Equivariant Minimal Immersions of $\mathcal{S}^{2}$ in $\mathcal{S}^{2 m}$, Trans. Amer. Math. Soc. 297 (1986), 105-124. MR849470 (87k:58061)

[11] D. Fox, Second Order Families of Coassociative 4-folds, Doctoral Dissertation, Duke University, 2005.

[12] D. Fox, Coassociative Cones that are Ruled by 2-Planes, Asian J. Math. 11 (2007), 535-554. MR2402937 (2009f:53072)

[13] R. Harvey, Spinors and Calibrations, Perspectives in Mathematics 9, Academic Press, Inc., Boston, Massachusetts, 1990. MR.1045637(91e:53056)

[14] R. Harvey and H. B. Lawson, Calibrated Geometries, Acta Math. 148 (1982), 47-152. MR666108 (85i:53058)

[15] O. Ikawa, T. Sakai and H. Tasaki, Weakly Reflective Submanifolds and Austere Submanifolds, J. Math. Soc. Japan 61 (2009), no. 2, 437-481. MR.2532897

[16] M. Ionel, Second Order Families of Special Lagrangian Submanifolds in $\mathbb{C}^{4}$, J. Differential Geom. 65 (2003), 211-272. MR2058262 (2005e:53075)

[17] D. D. Joyce, Ruled Special Lagrangian 3-folds in $\mathbb{C}^{3}$, Proc. London Math Soc. 85 (2002), 233-256. MR1901375 (2003i:53077)

[18] D. D. Joyce, Riemannian Holonomy Groups and Calibrated Geometry, Oxford Graduate Texts in Mathematics 12, Oxford University Press, Oxford, 2007. MR 2292510(2008a:53050)

[19] J. D. Lotay, Constructing Associative 3-folds by Evolution Equations, Comm. Anal. Geom. 13 (2005), 999-1037. MR2216150 (2007i:53052)

[20] J. D. Lotay, 2-Ruled Calibrated 4-folds in $\mathbb{R}^{7}$ and $\mathbb{R}^{8}$, J. London Math. Soc. 74 (2006), 219-243. MR2254562 (2008g:53061a)

[21] J. D. Lotay, Calibrated Submanifolds of $\mathbb{R}^{7}$ and $\mathbb{R}^{8}$ with Symmetries, Q. J. Math. 58 (2007), 53-70. MR 2305050 (2008a:53051)

[22] K. Mashimo, Homogeneous Totally Real Submanifolds of $\mathcal{S}^{6}$, Tsukuba J. Math. 9 (1985), 185-202. MR794670 (86j:53083)

[23] K. Sekigawa, Almost Complex Submanifolds of a 6-Dimensional Sphere, Kodai Math. J. 6 (1983), 174-185. MR702939 (84i:53054)

[24] L. Vrancken, Locally Symmetric Submanifolds of the Nearly Kähler $\mathcal{S}^{6}$, Algebras Groups Geom. 5 (1988), 369-394. MR.1104299 (91m:53020)

[25] L. Vrancken, Killing Vector Fields and Lagrangian Submanifolds of the Nearly Kähler $\mathcal{S}^{6}$, J. Math. Pures Appl. 77 (1998), 631-645. MR1645081 (99i:53064)

[26] L. Vrancken, Special Lagrangian Submanifolds of the Nearly Kähler 6-Sphere, Glasgow Math. J. 45 (2003), 415-426. MR2005344 (2004i:53067)

Department of Mathematics, Imperial College, London, SW7 2RH, United Kingdom E-mail address: jlotay@imperial.ac.uk 